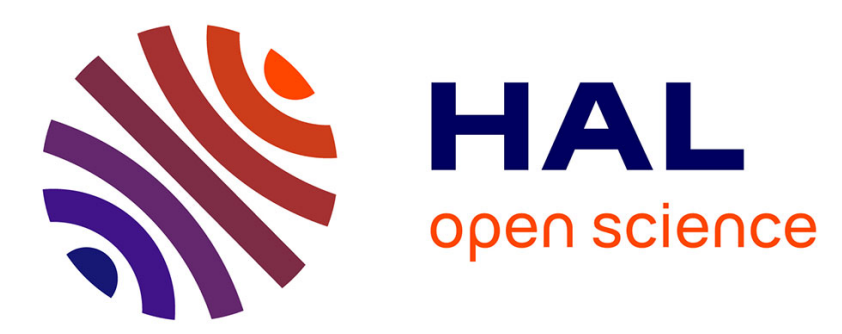

\title{
Numerical simulation of organized convection. Part I : model description and preliminary comparisons with squall line observations
}

Yves Pointin

\section{- To cite this version:}

Yves Pointin. Numerical simulation of organized convection. Part I : model description and preliminary comparisons with squall line observations. Journal of the Atmospheric Sciences, 1985. hal-01978001

\author{
HAL Id: hal-01978001 \\ https://hal.uca.fr/hal-01978001
}

Submitted on 11 Jun 2021

HAL is a multi-disciplinary open access archive for the deposit and dissemination of scientific research documents, whether they are published or not. The documents may come from teaching and research institutions in France or abroad, or from public or private research centers.
L'archive ouverte pluridisciplinaire HAL, est destinée au dépôt et à la diffusion de documents scientifiques de niveau recherche, publiés ou non, émanant des établissements d'enseignement et de recherche français ou étrangers, des laboratoires publics ou privés. 


\title{
Numerical Simulation of Organized Convection. Part I: Model Description and Preliminary Comparisons with Squall Line Observations
}

\author{
YVES POINTIN \\ L.A.M.P., Université de Clermont II, B.P. 45, 63170 Aubière, France
}

(Manuscript received 12 June 1984, in final form 15 October 1984)

ABSTRACT

\begin{abstract}
A numerical model designed for the simulations of mesoscale flows perturbed by deep convective clouds is discussed. It is based on the time dependent coupling between a three-dimensional nonhydrostatic mesoscale model and a quasi-one-dimensional cloud model. The evolution and motion of individual convective cells are simulated by the cloud model since they cannot be explicitly resolved by the mesoscale model. This implies that in cloudy areas each model simulates the time evolution of the same variables, at the same location and at the same time, under the influence of different processes (large-scale processes for the mesoscale model and microphysical processes for the cloud model). The comparison between the cloud and mesoscale rates of change leads to an evaluation of the coupling terms which transmit the cloud influences into the mesoscale model so that mesoscale fields are perturbed accordingly. For example, the nonhydrostatic pressure field reacts to the cloud development by building up an overpressure dome above the cloud top. On the other hand, the environmental conditions of each cell, including the vertical gradient of the nonhydrostatic pressure, represent the mesoscale influence on the cloud model. These conditions are deduced from the local values of the perturbed mesoscale fields at each cloud position.

A particular simulation is analyzed. Analysis reveals that the main characteristics of the flow perturbed by the convective cells are similar to those of a squall line deduced from ground station measurements. The typical signatures of the gust front are simulated, even though the magnitude of the perturbations are not all well simulated. The convective transport of horizontal momentum appears to be of fundamental importance to the organization process.
\end{abstract}

\section{Introduction}

In recent years, it has become increasingly recognized that deep convection is frequently organized into mesoscale structures such as multicell storms, squall lines, etc. The organization results from the mutual interactions between cloud elements, on a scale of hundreds of meters to kilometers, and the mesoscale flow, on a scale of tens of kilometers to hundreds of kilometers $[\beta$-mesoscale according to Orlanski's (1975) classification]: the convective transports of water, of energy and of momentum can modify the mesoscale flow in such a way that the latter forces the development of convective cells through low-level convergence or favorable nonhydrostatic pressure gradients. In order to study different mechanisms through which convective cells interact with the mesoscale flow (in a domain hundreds of kilometers wide), we developed a three-dimensional numerical model. This model is based on the mutual coupling between a nonhydrostatic three-dimensional mesoscale model and a time-dependent quasi-unidimensional cloud model. The general behavior of both models is very well-known and only the coupling procedure is somewhat new.

The peculiarities of such a mesoscale model stem from the fact that the simulation domain must be large enough $(160 \mathrm{~km} \times 160 \mathrm{~km}$ in our case $)$ to simulate mesoscale perturbations but it also should resolve all motions and physical processes of cloud scales. However, the limited speed of modern computers implies that the number of grid points must be limited so that the grid size must be of several kilometers $(5 \mathrm{~km}$ in our case). With such a grid size, the convective motions cannot be explicitly resolved on the mesoscale grid as Yau and Michaud (1982) or Wilhelmson and Chen (1982) have done in smaller domains with a grid size of $1-1.5 \mathrm{~km}$. It is true that Rosenthal (1979) obtained interesting results in cyclone simulations with a model in which condensation is completely resolved on the mesoscale grid with a mesh size of $20 \mathrm{~km}$. However, for organized deep convection, such as in squall lines, the localized effects of the downdrafts generated by precipitation loading and evaporational cooling are of fundamental importance in the dynamics of these systems (Zipser, 1977; Moncrieff, 1981). Also, the large speed of the vertical convective motions, including precipitation fallout, must be obtained in the model. These phenomena cannot be explicitly resolved and simulated even with a $5 \mathrm{~km}$ grid model. Therefore, the intensity, location and time evolution of the convective sources and sinks must be estimated in a different way. The convective parameterizations (Kuo, 1974; Arakawa 
and Schubert, 1974; Anthes, 1977; Geleyn et al., 1982), which are widely used in synoptic models in order to provide such estimates of the convective influences, are based on a quasi-equilibrium hypothesis requiring that many cells evolve substantially during the synoptic time step and in each synoptic grid mesh. This hypothesis cannot hold in a model with a grid size of $5 \mathrm{~km}$ and a corresponding time step of $20 \mathrm{~s}$. Therefore, a new procedure is required.

There are already several models based on the coupling between a one-dimensional cloud model and a three-dimensional mesoscale model (Kreitzberg and Perkey, 1976; Anthes, 1977; Fritsch and Chappell, $1980 \mathrm{a}, \mathrm{b})$, but these mesoscale models are all hydrostatic models with a grid size greater than $20 \mathrm{~km}$ and the cloud models are all stationary Lagrangian models. Our model differs from these models in some important physical features. The mesoscale model is fully compressible with a grid size of $5 \mathrm{~km}$ and a time step of $20 \mathrm{~s}$ and is such that nonhydrostatic pressure gradients can be induced by the cloud cells and influence their development. These spatial and temporal resolutions should be appropriate to the simulation of the main characteristics of the flow that develops in the active part . of a squall line, for example. The cloud model is time-dependent, quasione-dimensional (Wang, 1983), and can simulate the rapid increase of the cloud height $\left(10\right.$ to $\left.20 \mathrm{~m} \mathrm{~s}^{-1}\right)$ and the fast evolution, due to microphysical processes including precipitation fallout, which is badly simulated with a stationary model. Furthermore, precipitation unloading by the wind shear and vertical transport of horizontal momentum are simulated by the cloud model. Fritsch and Chappell (1980a) discussed the important effects of these two processes.

The coupling procedure between the two timedependent models is based on the fact that the two models are integrated in parallel at the same time. The cloud development is simulated by the cloud model as the cell moves across the mesoscale domain. During its whole life, the local conditions that it experiences on its outside (thermodynamical profiles) and the forcing that it undergoes (nonhydrostatic pressure gradient) are evaluated, at each time step, from the local values of the mesoscale fields at the instantaneous cloud position. As the cell evolves, it locally influences the mesoscale environment through the vertical fluxes of mass, momentum and energy that it generates. The effects of these convective fluxes on the mesoscale fields are quantified by convective forcing terms which are introduced in the mesoscale equations as local sources or sinks of mass, momentum and energy. These convective forcing terms are evaluated, using a conservation principle, from the comparison of the time evolutions of the cloud and of the mesoscale variables under the influence of specific processes (microphysical processes and largescale processes).
This comparison procedure avoids defining the convective forcing terms from the vertical profile of in-cloud parameters by using somewhat arbitrary principles, such as detrainment at the cloud top (Arakawa and Schubert, 1974), mixing of the cloud with its environment (Kuo, 1974), turbulent mixing (Anthes, 1977) or direct influence of the sources and sinks induced by in-cloud condensation (Fritsch and Chappell, 1980a,b): Our method is not, however, free from arbitrariness since a new cell is created whenever the mesoscale flow satisfies some criteria (e.g., instability, convergence) at some location which becomes the initial position of the new cell. Therefore, the convective activity that develops in the domain is very dependent upon these creation criteria and upon the total number of cells that can exist at any one time. In some cases, such as squall lines, the preferred location for the creation of new cells is better known. However, the comparison between the simulation results and experimental data may still be difficult because the timing for the cell creations is still largely unknown.

The mesoscale model is schematically described in Section 2, the cloud model in Section 3 and the coupling procedure in Section 4. A complete description of both models can be found in Pointin (1984b). Simulation results are presented in Section 5 and compared with some experimental features of a squall line observed during the French-Ivory Coast COPT 81 experiment (Sommeria and Testud, 1984).

\section{Mesoscale model}

\section{a. Basic equations}

The physics of the three-dimensional nonhydrostatic model are not very elaborate since the microphysical processes and the convective vertical motions are explicitly simulated by the cloud model and their effects are introduced in the mesoscale equations through convective forcing terms. This model integrates the primitive equations in fully compressible form with a semiimplicit method developed by Tapp and White (1976, hereafter TW).

The basic variables are:

u the velocity vector

$P$ the total pressure

$T$ the gas temperature

$\rho$ the total density (dry air and water vapor)

$q_{m}$ the water vapor deficit (Kessler, 1969) which indicates when condensation or evaporation occurs by passing through zero.

We use the definition

$$
q_{m}=\left\{\begin{array}{lll}
q_{v}-q_{s} & q_{m} \leqslant 0 & \text { undersaturation } \\
q_{c} & q_{m}>0 & \text { condensation }
\end{array}\right.
$$


where $q_{v}, q_{s}$ and $q_{c}$ are the mass per unit volume of the water vapor, of the saturated water vapor and of the liquid water respectively. The equations of the mesoscale model are written in terms of the perturbation pressure $P^{\prime}$ which is defined with respect to a hydrostatic pressure profile $\bar{P}(z)$ deduced from the undisturbed temperature profile $\bar{T}(z)$. Also,

$$
\begin{gathered}
P^{\prime}(x, y, z, t)=P(x, y, z, t)-\bar{P}(z), \\
\frac{\partial \bar{P}(z)}{\partial z}=-\bar{\rho}(z) g, \\
\bar{P}(z)=R \bar{\rho}(z) \bar{T}(z) / M_{d},
\end{gathered}
$$

where $R$ is the perfect gas constant and $M_{d}$ the molecular mass of dry air.

The mesoscale equations include the momentum equation:

$$
\begin{aligned}
\frac{\partial \mathbf{u}}{\partial t}+\mathbf{u} \cdot \nabla \mathbf{u}=- & \nabla P^{\prime} / \bar{\rho}-g \mathbf{k}(1-\bar{\rho} / \rho) \\
& +\nabla P^{\prime}(\rho-\bar{\rho}) /(\rho \bar{\rho})-\mathbf{f} \times \mathbf{u}+\mathbf{F},
\end{aligned}
$$

where $\mathbf{k}$ is the vertical vector and $\mathbf{f}$ the Coriolis vector. The force term $F$ includes the turbulent Reynolds stresses and the convective forcing body force $\mathbf{F}_{c}$ which is evaluated in Section 4 .

The pressure tendency equation replaces the continuity equation and reads:

$$
\begin{aligned}
\frac{\partial P^{\prime}}{\partial t}+\text { 边 } \cdot \nabla P^{\prime}=w \bar{\rho} g- & \gamma \bar{P} \operatorname{divu} \\
& -\gamma P^{\prime} \operatorname{divu}+\rho(\gamma-1) Q,
\end{aligned}
$$

where $\gamma$ is the ratio of the specific heats of dry air and $Q$ is the heat source which appears also in the thermodynamic equation:

$$
\frac{\partial T}{\partial t}+\mathbf{u} \cdot \nabla T=Q-\left(\frac{\partial P^{\prime}}{\partial t}+\mathbf{u} \cdot \nabla P^{\prime}-w \bar{\rho} g\right) / C_{p d},
$$

where $C_{p d}$ is the specific heat at constant pressure of dry air. The heat source $Q$ also includes the convective forcing heat source $Q_{c}$. The water vapor continuity equation reads:

$$
\begin{aligned}
\frac{\partial q_{m}}{\partial t}+\operatorname{div}\left[\left(q_{m}+q_{s}\right) \mathbf{u}\right] & \\
& =-\left(\frac{\partial q_{s}}{\partial T}\right) \frac{\partial T}{\partial t}-\left(\frac{\partial q_{s}}{\partial P}\right) \frac{\partial P}{\partial t}+S_{c},
\end{aligned}
$$

where $S_{c}$ is the convective forcing water vapor source. The influence of water vapor and liquid water on the thermodynamical equation can be easily taken into account by using the wet equivalent enthalpy proposed by Pointin (1984a).

\section{b. Main characteristics}

The main characteristics of the model are as follows:

1) The time discretization is done between time levels $t_{n}$ and $t_{n+1}$ as

$$
\frac{\partial X}{\partial t} \approx \frac{X^{n+1}-X^{n}}{\Delta t}
$$

where $X$ is any variable and $\Delta t$ is the mesoscale time step. This discretization is suggested by the evaluation of the convective forcing terms as explained in Section 4 and replaces the "leap-fog" time discretization used in $T W$. Although this time discretization may lead to some instabilities when centered differencing is used in the advection terms, these instabilities have been practically eliminated in the described simulations by using the following differencing:

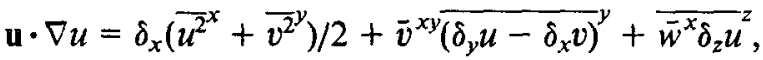

$$
\begin{aligned}
& \mathbf{u} \cdot \nabla w={\overline{\bar{u}^{z} \delta_{x} w}}^{x}+{\overline{\bar{v}^{z} \delta_{y} w}}^{y}+{\overline{\bar{w}^{z} \delta_{z} w}}^{z} \text {, }
\end{aligned}
$$

where

$$
\begin{aligned}
\delta_{x} X & =\left(X_{i+1 / 2, j, k}-X_{i-1 / 2, j, k}\right) / \Delta x, \\
\bar{X}^{x} & =\left(X_{i+1 / 2, j, k}+X_{i-1 / 2, j, k}\right) / 2,
\end{aligned}
$$

2) The semi-implicit method of integration requires that the first two terms in the right-hand sides of (5) and (6) be evaluated as one mean value between the value at time level $n$ and that at time level $n$ +1 . This results into a set of two coupled discretized equations which are transformed into a Helmholtztype equation as described in the Appendix.

3) The grid is staggered in all directions so that all of the variables are defined at the grid points except for the velocity components, which are located half a grid length from the grid points in their respective direction (C grid of Arakawa and Lamb, 1977). Grid sizes are constant in the horizontal directions $(\Delta x$ $=\Delta y=5 \mathrm{~km}$ ) but increase with height in the vertical direction $(0.6 \mathrm{~km}<\Delta k<2.1 \mathrm{~km})$.

4) Spatial derivatives are evaluated from centered three-point formulae, which are second-order except for the second vertical derivatives, which are firstorder due to the increasing grid thickness.

5) The model is bounded below by a constant flux layer in which the turbulent fluxes are determined from Monin-Obukhov similarity theory. The stability functions are given by Nickerson and Smiley (1975), the ground values are kept constant and the vertical profiles of the heat and momentum eddy diffusion coefficients inside the boundary layer, $1 \mathrm{~km}$ deep, are given by O'Brien (1970).

6) Turbulent Reynolds stresses due to subgrid motions, excluding the convective motions simulated by the cloud model, are defined with a constant value of horizontal eddy diffusion coefficients above the boundary layer. For simplicity, this form of the 
turbulent Reynolds stresses is also used at grid points covered by convective cells, although the latter give rise to most of the mesoscale subgrid motions in those points. It is not clear what terms must be added to the convection forcing terms, there, in order to represent the turbulent Reynolds stresses in the mesoscale equations.

7) The lateral boundary condition for the normal velocity is specified according to Orlanski (1976) as

$$
\frac{\partial u_{n}}{\partial t}=-\left(c+u_{n}\right) \mathbf{n} \cdot \nabla u_{n}
$$

where $\mathbf{n}$ is the outward normal vector and $u_{n}=\mathbf{u} \cdot \mathbf{n}$ the outward component of the velocity vector. The term $c$ is a constant wave velocity $\left(c=20 \mathrm{~m}^{\prime} \mathrm{s}^{-1}\right)$.

8) At the upper boundary, the evolution of the vertical velocity is adopted from Tripoli and Cotton (1982) as

$$
\frac{\partial w}{\partial t}=-(c+w) \operatorname{div}(\rho \mathbf{u}) / \rho,
$$

and at the bottom boundary, the vertical velocity is set to zero since the model does not include topography.

9) The lateral, upper and lower boundary conditions for the other variables, except the pressure, are such that the normal advection terms are set to zero for incoming fluid particles $\left(u_{n}<0\right.$ or $\left.w_{3 / 2}>0\right)$ and are evaluated from an "upstream" formula for outgoing fluid particles $\left(u_{n}>0\right.$ or $\left.w_{3 / 2}<0\right)$.

10) The boundary condition for the pressure is deduced from the discretized pressure tendency equation (6) written at the boundary and with the normal advection term set to zero. In order to keep the form of the discretized Helmholtz equation, derived in the Appendix, an extra point is introduced outside the domain. The value of the pressure at this extra point is deduced from the discretized momentum equation (5) written at the boundary but with the new value of the normal velocity $\left(u_{n}^{n+1}\right)$ evaluated from (10) or (11). This leads to a Neumann-type boundary condition for the Helmholtz equation. This boundary condition preserves the balance between the pressure and the velocity fields through sound waves-balance that is fundamentally important for this model with the grid size and the time step of $5 \mathrm{~km}$ and $20 \mathrm{~s}$ respectively.

Simulations show that computational oscillations are minimum while the period of most sound waves is increased by time truncation to $4 \Delta t(T W)$. As in other fully compressible models (Tripoli and Cotton, 1982), the open boundary conditions lead to a slow change in the mean total pressure in the domain. Furthermore, the upper boundary condition (11) produces a spurious increase of the absolute values of the vertical velocity at the top of the domain (17.3 $\mathrm{km}$ ) which remain small up to $\mathbf{4 0} \mathrm{min}$ and increase almost exponentially towards the end of the simulation.

\section{Cloud model}

This model simulates the development of each convective cell evolving in the mesoscale domain. It is, in many respects, similar to the model developed independently by Wang (1983). Only the main features, including those which are essential in the coupling procedure with the mesoscale model, are described here. Details can be found in Pointin (1984b).

Figure 1 shows that the convective cell is made of three concentric cylinders, described by an Eulerian grid and topped by a Lagrangian hemispheric dome which rises rapidly during the cloud development. The internal cylinder (of index $\alpha=1$ ) represents the active part of the cloud in which the main updraft is generated. The localized downdraft, which is sometimes induced by precipitation loading and evaporational cooling, can develop in the intermediate cylinder $(\alpha=2)$. The external cylinder $(\alpha=3)$ represents the close environment of the cloud, in which a weak large-scale compensating motion can develop; it also transmits the local changes in environmental conditions to the cloud.

The basic variables, which are mean values over each annulus cross section, are only functions of the vertical coordinate and of time and include:

$$
\begin{array}{ll}
w_{\alpha} & \begin{array}{l}
\text { the vertical component of the veloc- } \\
\text { ity, }
\end{array} \\
T_{V \alpha} & \begin{array}{l}
\text { the virtual temperature, } \\
\text { the total density, }
\end{array} \\
\rho_{\alpha} & \begin{array}{l}
\text { the water vapor deficit and the mass } \\
q_{m \alpha}, q_{r \alpha},
\end{array} \\
q_{i \alpha}, q_{h \alpha} & \begin{array}{l}
\text { per unit volume of the rain drops, } \\
\text { of the ice particles and of the grau- } \\
\text { pel respectively, }
\end{array} \\
E_{c \alpha}, E_{T \alpha} & \begin{array}{l}
\text { the kinetic and the thermal turbu- } \\
\text { lent energies respectively as in Lo- }
\end{array} \\
R_{\alpha} & \begin{array}{l}
\text { pez (1973), } \\
\text { the radius of each cylinder, } \\
\text { the horizontal velocity vector. }
\end{array}
\end{array}
$$

The same variables with the index $T$ instead of $\alpha$ denote the values in the Lagrangian cloud top. Other variables are needed to describe the cloud cell. They are only functions of time and include:

$$
\begin{aligned}
& Z_{B}, Z_{T} \begin{array}{l}
\text { the height of the cloud base and cloud } \\
\text { top respectively, } \\
\text { the position of the mean axis of the }
\end{array} \\
& \mathbf{X}_{N} \quad \begin{array}{l}
N \text { th cell, } \\
\text { the mean horizontal velocity of the } \\
N \text { th cell. }
\end{array} \\
& \mathbf{u}_{N}
\end{aligned}
$$

The main characteristics of the cloud model are as follows: 


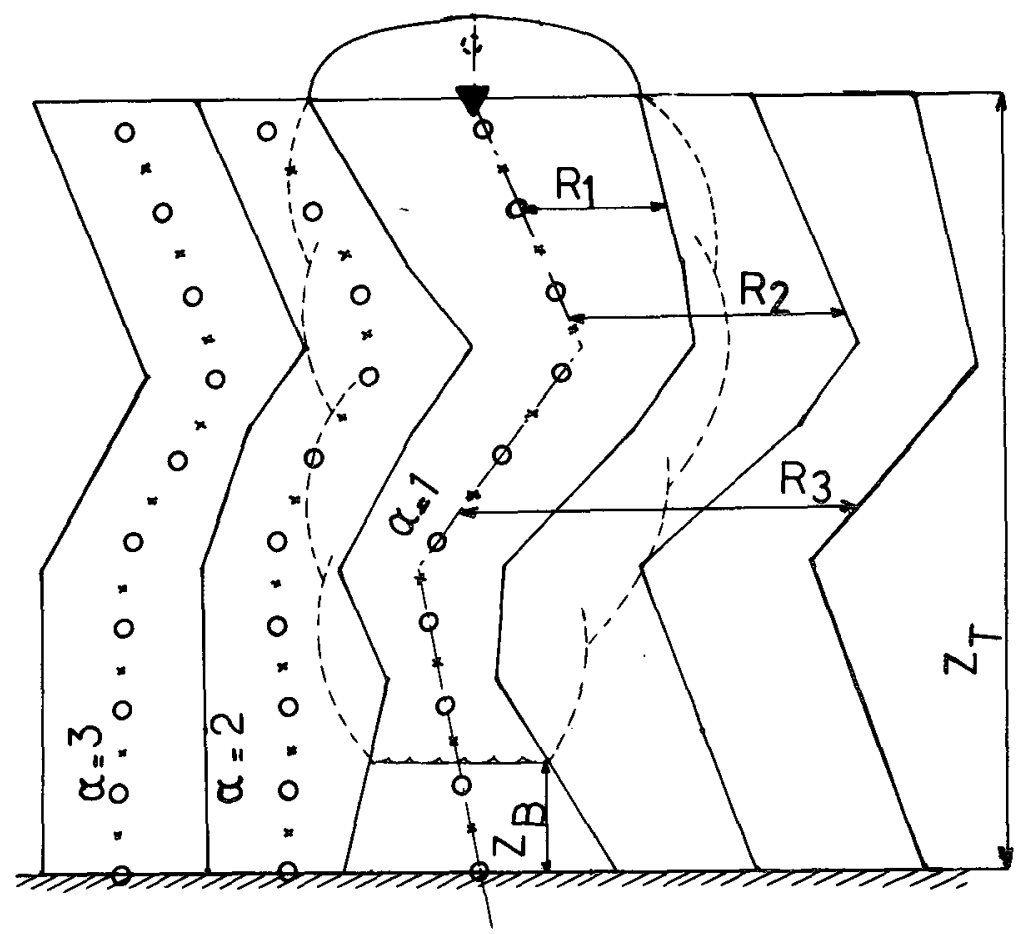

FIG. 1. Sketch of the one-dimensional cloud with a slanting axis and variable radius. The location of the Lagrangian variables of index $T$ is shown by the solid triangle. Circles mark where the Eulerian variables are situated in each cylinder. The location of the nonhydrostatic pressure is marked by a cross.

1) Bulk microphysical equations are very similar to those used by Ogura and Takahashi (1971), Wisner $e t$ al. (1972) or Cotton and Tripoli (1978).

2) Entrainment and detrainment between one annulus and another are defined in term of a turbulent entrainment velocity

$$
V_{\alpha}=\delta E_{c \alpha}^{1 / 2},
$$

where $\delta=0.3$. This implies that each evolution equation for any intensive variable $X^{\prime}\left(=T_{V}, q_{m} / \rho, E_{c}\right.$, $\mathbf{u}$, and so on) includes the advection-entrainment term as:

$$
\begin{aligned}
\left(\frac{\partial X_{\alpha}^{\prime}}{\partial t}\right)_{\mathrm{adv}} & =-w_{\alpha} \frac{\partial X_{\alpha}^{\prime}}{\partial z}+C_{\alpha} V_{\alpha} \rho_{\alpha+1}\left(X_{\alpha+1}^{\prime}-X_{\alpha}^{\prime}\right) / \rho_{\alpha} \\
& +C_{\alpha-1} V_{\alpha} \rho_{\alpha-1}\left(X_{\alpha-1}^{\prime}-X_{\alpha}^{\prime}\right) S_{\alpha-1} /\left(\rho_{\alpha} S_{\alpha}\right),
\end{aligned}
$$

where $S_{\alpha}=\pi\left(R_{\alpha}{ }^{2}-R_{\alpha-1}{ }^{2}\right)$ is the cross section of the annulus between the radius $R_{\alpha}$ and $R_{\alpha-1}\left(R_{0}=0\right)$. The $C_{\alpha}$ represents the ratio of the lateral surface $A_{\alpha}$ per unit height to the cross section $S_{\alpha}$ (Wisner et al., 1972):

$$
A_{\alpha}=C_{\alpha} S_{\alpha}=2 \pi R_{\alpha}\left[1+\left(\frac{\partial R_{\alpha}}{\partial z}\right)^{2}\right]^{1 / 2} \text {. }
$$

3) The evolution of the turbulent energies appearing in (12) is predicted with highly parameterized turbulent equations obtained following Lopez (1973).
4) Besides turbulent entrainment, precipitating particles (rain drops or graupel) can fall through the lateral surface either when the radius increases with height or when the axis of the cylinder makes the angle with the vertical axis (Fig. 2). This angle can be deduced from the vertical gradient of the horizontal distance that the cloud has covered, at each altitude, from its creation at time $t_{i}$ :

$$
\operatorname{tg} \beta_{(z, t)}=\left\|\frac{\partial}{\partial z} \int_{t_{i}}^{t}\left(\mathbf{u}_{1}-\mathbf{u}_{N}\right) d t\right\| .
$$

5) the Lagrangian cloud top rises according to

$$
\frac{d Z_{T}}{d t}=w_{T}
$$

where $w_{T}$ is the value of the vertical velocity at the cloud top. As it rises, the cloud top passes over Eulerian grid points which are then included in the cylindrical cloud core. The value of each variable at this new grid point is deduced from the value of the corresponding cloud top variable at the crossing time. The Lagrangian equations for the cloud top variables include the effect of lateral entrainment, but also of vertical advection which takes the form of the first term in the right-hand side of the advection-entrainment rate of change for any intensive variable $X^{\prime}$ : 


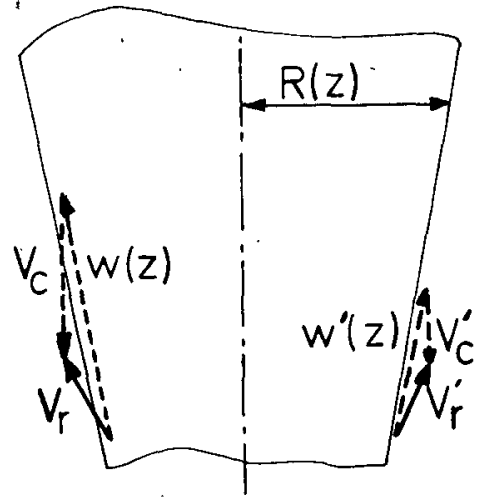

a

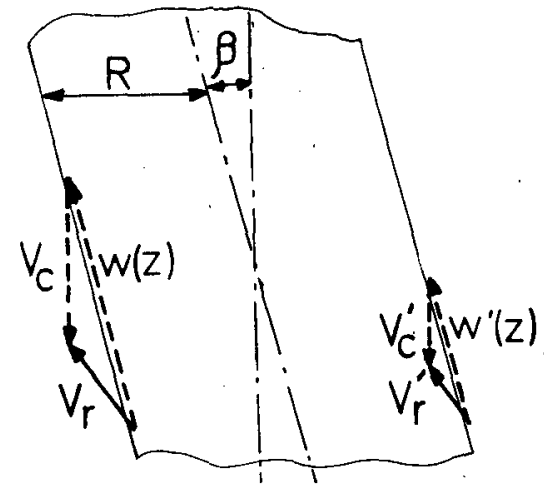

b

FIG. 2. Schematic vertical section of one of the cloud cylinders showing how precipitating particles can pass from one cylinder to another when a) the radius is function of height, b) the axis of the cylinder is slanted by an angle $\beta . w$ is the air velocity, $V_{c}$ the terminal velocity of the particle and $V_{r}$ their absolute velocity: Unprimed variables refer to internal values, primed ones to external values.

$$
\begin{array}{r}
\left(\frac{d X^{\prime}}{d t}\right)_{\mathrm{adv}}=3 \rho_{1}\left(w_{1}-w_{T}\right)^{+} /\left(2 a R_{T} \rho_{T}\right)\left(X_{1}^{\prime}-X_{T}^{\prime}\right) \\
+3 V_{T} \rho_{3}\left(X_{3}^{\prime}-X_{T}^{\prime}\right) /\left(2 \rho_{T} R_{T}\right),
\end{array}
$$

where $(\cdot)^{+}=\max (\cdot, 0)$ vanishes if the cloud top rises faster than the speed of the air in the underneath core and $a$ is a flatness coefficient $(a=0.8)$.

6) The horizontal motion of the cell is deduced from

$$
\frac{d \mathbf{X}_{N}}{d t}=\mathbf{u}_{N}
$$

where the mean horizontal velocity of the $N$ th cell is given by

$$
\mathbf{u}_{N}=\int_{0}^{Z_{T}} \rho_{1} R_{1}{ }^{2} \mathbf{u}_{1} d z / \int_{0}^{Z_{T}} \rho_{1} R_{1}{ }^{2} d z .
$$

7) At the altitude $z$, the horizontal velocity of the horizontal section of the cell results from vertical advection, entrainment of horizontal momentum and the action of the drag force $\mathbf{F}_{f}$ exerted by the other cylinders:

$$
\mathbf{F}_{f}=C_{D}\left\|\mathbf{u}_{n}-\mathbf{u}_{\mathrm{ex}}\right\|\left(\mathbf{u}_{n}-\mathbf{u}_{\mathrm{ex}}\right) R \rho_{\mathrm{ex}},
$$

where $u_{n}$ is the velocity of the annulus of external radius $R$ and the subscript ex refers to the external (or internal) flow. The value of the friction coefficient $C_{D}$ is 0.3 . Therefore, the horizontal momentum equation reads

$$
\frac{\partial \mathbf{u}_{\alpha}}{\partial t}=\left(\frac{\partial u}{\partial t}\right)_{\mathrm{adv}}+\left(\frac{\partial \mathbf{u}}{\partial t}\right)_{\mathrm{fr}}+\frac{\nu \partial^{2} \mathbf{u}}{\partial z^{2}}
$$

where the rate of change with subscript adv is obtained from (13) [or (17) for the cloud top] and where the friction term is given by

$$
\begin{aligned}
& \left(\frac{\partial \mathbf{u}_{\alpha}}{\partial t}\right)_{\mathrm{fr}} \\
& =-C_{D}\left[\left\|\dot{u}_{\alpha}-\mathbf{u}_{\alpha-1}\right\|\left(\mathbf{u}_{\alpha}^{\prime}-\mathbf{u}_{\alpha-1}\right) R_{\alpha-1} \rho_{\alpha-1}\right. \\
& \left.\quad+\left\|\mathbf{u}_{\alpha}-\mathbf{u}_{\alpha+1}\right\|\left(\mathbf{u}_{\alpha}-\mathbf{u}_{\alpha+1}\right) R_{\alpha} \dot{\rho}_{\alpha+1}\right] /\left(S_{\alpha} \rho_{\alpha}\right) .
\end{aligned}
$$

These equations define the vertical transport of horizontal momentum by the convective cells. This transport is shown in Section 5 to be fundamentally important to the convective organization process.

8) The bottom boundary conditions must be specified according to the large-scale flow so that the cloud does not constitute an uncontrolled source of mass, momentum or energy. Therefore, the vertical mass flux in the cloud cross section must be equal to the mesoscale mass flux at the cloud base, namely

$$
\sum_{\alpha} S_{\alpha} \rho_{\alpha} w_{\alpha}=-\left(\sum_{\alpha} S_{\alpha}\right)(\operatorname{divu})_{s} \bar{\rho} Z_{B}
$$

where $\bar{\rho}$ is a mean density, $Z_{B}$ the height of the cloud base and (divu) is a mean value of the mesoscale divergence of the horizontal velocity field at the ground, over the cross section of the cell. The value of the vertical velocity at the bottom of the cloud (Fig. 1) in the internal cylinder is deduced from (23), in which the value in the other cylinders is either obtained from the general equation of motion with upstream advection derivatives when this velocity value is negative, or is taken as some fraction of the vertical velocity value in the internal cylinder otherwise. The values of the temperature and of the water vapor deficit at the bottom are deduced from similar budget equations. The advection terms for the other variables are either set to zero when the vertical velocity is positive or are computed with an upstream formula otherwise. 
9) The vertical momentum equation does not include a virtual mass coefficient, but a nonhydrostatic pressure gradient computed from the mesoscale model (as will be explained) modifies the acceleration due to buoyancy effects.

\section{Coupling algorithm \\ a. Principle}

As explained in Section 1, each convective cell evolving in the mesoscale model is simulated by a quasi-one-dimensional cloud model. As it develops, the cell moves across the domain and therefore meets different environmental conditions. Furthermore, it influences the mesoscale dynamical and thermodynamical fields through vertical fluxes of mass, energy and momentum. This influence is parameterized by convective forcing terms which are added as sources or sinks in the equations of the mesoscale model.

The coupling algorithm is illustrated in the temporal diagram in Fig. 3. The cloud model is schematized at the top, the mesoscale model at the bottom, while the coupling terms (environment of the cell, convective forcing terms) are in the middle lozenges. The evaluation of these terms is described in the following sections and the different steps of the coupling algorithm are as follows:

1) The values of the mesoscale variables being defined at time $t_{n}=n \Delta t$, the $N$ th cell moves to its new position according to (18). The values of the variables in the external cylinder of this cell are evaluated from the local values of the mesoscale variables and from the values of the variables in the other two cylinders as will be explained. The nonhydrostatic vertical pressure gradient and the mean value of the divergence of the horizontal wind at the ground are also evaluated during this step.

2) For each cell, the cloud model is integrated with a variable time step $\delta t \ll \Delta t$, while the forcing terms (pressure gradient and divergence) are kept constant. The last time step is chosen so that the value of the final time corresponds to the mesoscale time $t_{n+1}$ $=(n+1) \Delta t$.

3) The convective forcing terms are evaluated from the time evolution of the cloud variables and from the evolution of the mesoscale variables under some processes, as explained in Section 4c.

4) The mesoscale equations, in which the convective forcing terms are kept constant, are integrated from time $t_{n}$ to time $t_{n+1}$ by the semi-implicit method.

5) Integration proceeds by going back to step 1.

This description shows that the cloud model and the mesoscale model are integrated in parallel and that, during each time step $\Delta t=20 \mathrm{~s}$, a coupling term, evaluated from one model, modifies the evolution predicted by the other one. Therefore, the time evolution of the variables in both models is of fundamental importance in the coupling algorithm.

The evaluation of these coupling terms is based on the geometrical position of the cell in the mesoscale grid. This position is characterized by the values $A_{i j \alpha}$ of the area that the $\alpha$ th cylinder of the $N$ th cell covers, at time $t_{n}$ and at altitude $z_{k}$, on the grid mesh centered at the point of coordinates $\left(x_{i}, y_{j}, z_{k}\right)$. The

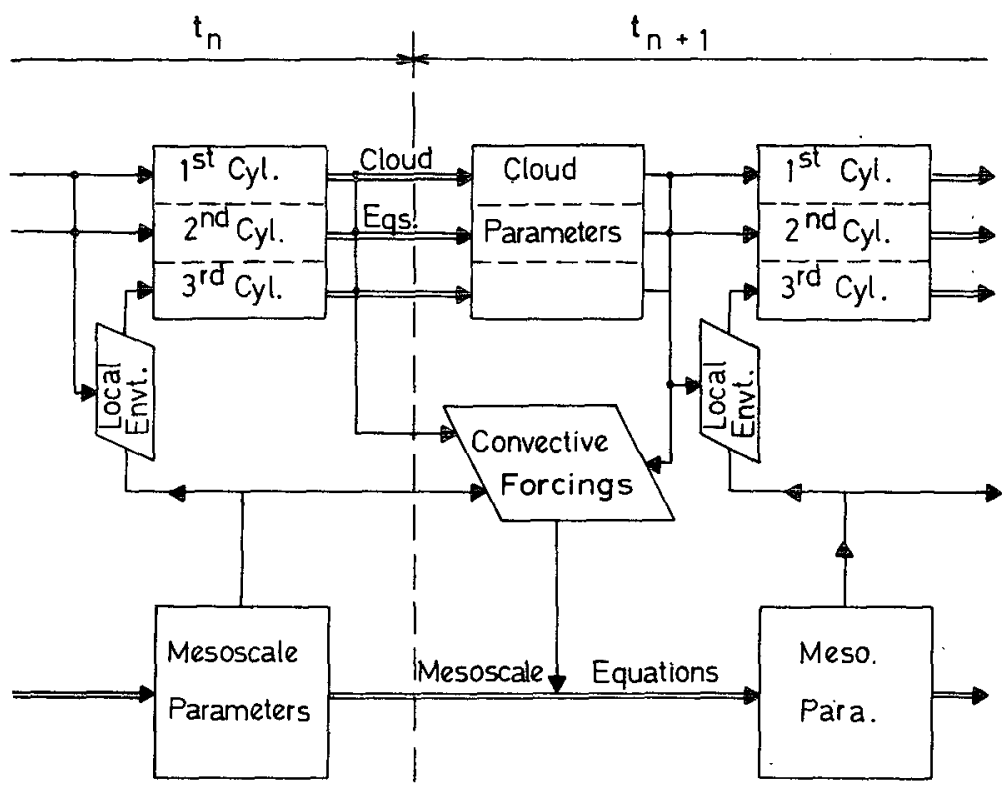

FIG. 3. Block diagram of the coupling procedure between the cloud model (rectangles at the top) and the mesoscale model (rectangles at the bottom). The coupling terms are in the middle lozenges and time runs from left to right. 
dependence of $A_{i j \alpha}$ upon the cell number $N$, time $t_{n}$, and upon the altitude $z_{k}$ is omitted in the following. The definition of these areas is illustrated in Fig. 4, which shows a horizontal cross section of the mesoscale grid in which a cloud cell is situated.

\section{b. Evaluation of the environment of the cell}

The value of each variable in the external cylinder is not just obtained from mean local values of the mesoscale fields, as is usually done; its evaluation is based on a conservation principle applied to the averaged value of this variable over the horizontal cross section of the external cylinder of each cell. Namely, the horizontal integral over this section of any variable $X$, at the altitude $z_{k}$ and at time $t_{n}$, can be expressed either in terms of the values $X_{i j k}$ it takes in the mesoscale model at the grid point situated at the coordinates $\left(x_{i}, y_{j}, z_{k}\right)$, or in terms of the values $X_{\alpha}$ it takes in the $\alpha$ th cylinder of the $N$ th cell:

$$
\begin{aligned}
\int_{\text {meso }} X d s & \equiv \sum_{i} \sum_{j} X_{i j k}\left(A_{i j 1}+A_{i j 2}+A_{i j 3}\right) \\
& =\sum_{\alpha} S_{\alpha} X_{\alpha} \equiv \int_{\text {cloud }} X d s .
\end{aligned}
$$

The middle equality is used to evaluate the value $X_{3}$ of the variable $X$ in the external cylinder of the cell during step 1 of the coupling algorithm. This value depends on the values $X_{i j k}$ of the mesoscale variable but also on the values $X_{1}$ and $X_{2}$ of the variable in the other two cylinders. This procedure implies a partial compensation for a high positive value in the internal cylinder by a low negative value in the external cylinder, provided that the integrated mean value in the mesoscale model remains zero. In fact, this last value is likely to increase in response to the convective forcing terms induced by the large positive value in the internal cylinder.

When some variables are defined in one model but not in the other (24) is useless. This is the case for the nonhydrostatic pressure $P_{\alpha}^{\prime}$ in the $\alpha$ th cylinder, which is obtained as a mean value from the mesoscale values $P_{i j k}^{\prime}$ as

$$
P_{\alpha}^{\prime}=\sum_{i} \sum_{j} A_{i j \alpha} P_{i j k}^{\prime} / \sum_{i} \sum_{j} A_{i j \alpha} .
$$

A similar equality gives the value (divu) $)_{s}$ of the mean horizontal divergence at the ground. On the other hand, the values $E_{c 3}$ and $E_{T 3}$ of the turbulent intensities in the external cylinders are diagnosed from the mean values of the mesoscale flow fields using turbulent equations derived by Sommeria (1976) assuming that horizontal gradients of thermodynamic quantities vanish.

\section{c. Evaluation of the convective forcing terms}

In previous works, the convective forcing terms which in the mesoscale equations influence the evo-

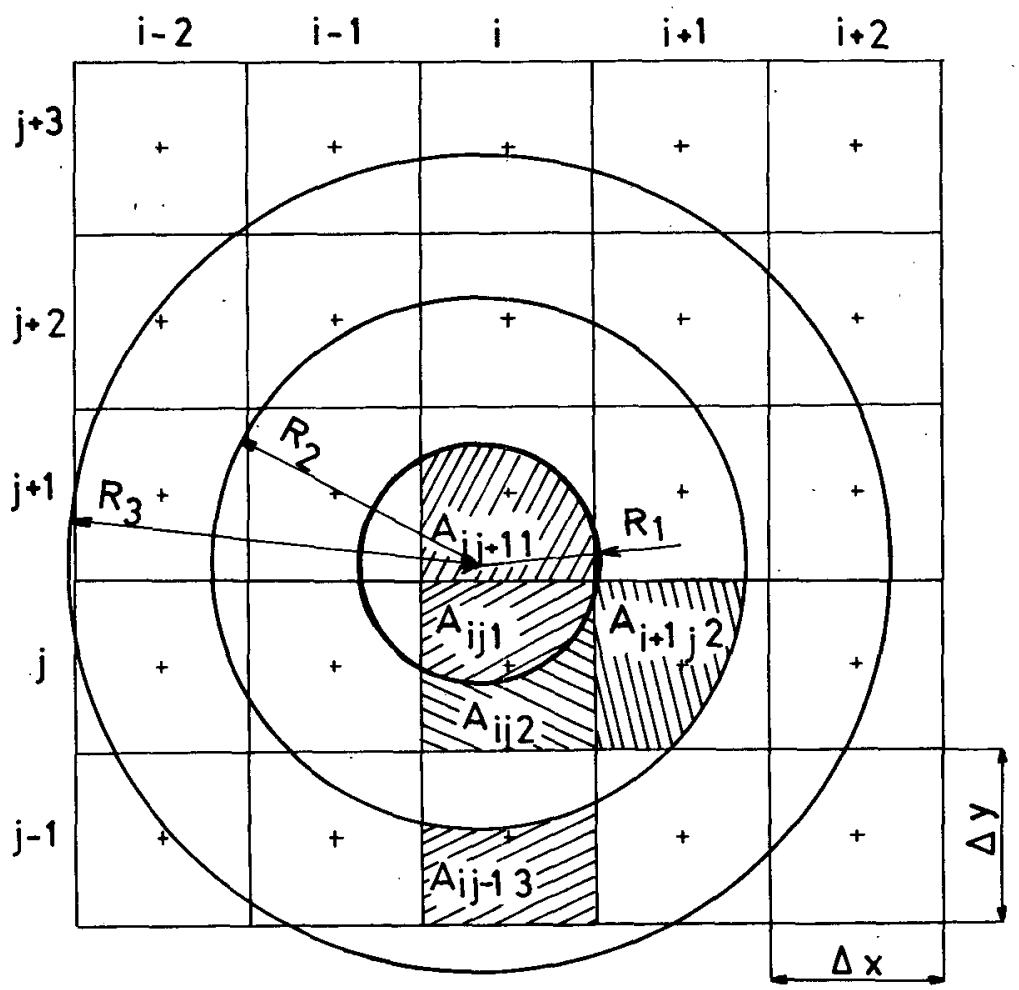

FiG. 4. Horizontal cross section at time $t_{n}$ and altitude $z_{k}$ of the mesoscale domain in which a cell is centered at $\left(X_{N}, Y_{N}\right)$ in the $(i, j+1)$ grid mesh. This section shows some of the areas $A_{i j \alpha}$ that are used in the evaluation of the coupling terms. 
lution of the mesoscale fields are evaluated by formulae based on different principles: detrainment at the cloud top (Arakawa and Schubert, 1974); mixing of the cloud in its environment (Kuo, 1974); turbulent fluxes (Anthes, 1977); or direct forcing by the sources and sinks induced by in-cloud condensation (Fritsch and Chappell, 1980a,b). Our method takes advantage of both models simulating the time evolution of the same variables, at the same time and location, under the influence of different processes (large-scale processes and cloud-scale processes). In the mesoscale model, the complete partial time derivative of any variable $X$ can be split, according to the scale. of processes inducing this temporal change, as

$$
\left(\frac{\partial X}{\partial t}\right)_{i j k}=\left(\frac{\partial X}{\partial t}\right)_{\mathrm{LS}}+\left(\frac{\partial X}{\partial t}\right)_{\mathrm{v}}+\left(\frac{\partial X}{\partial t}\right)_{\mathrm{c}}
$$

where the index $c$ refers to cloud-scale processes which are not explicitly simulated by the mesoscale model. The introduction of the convective forcing terms $F_{X}$ in the mesoscale equations can bring in this model the effects of the cloud scale processes, provided $F_{X}$ is defined by

$$
F_{X}=(\partial X / \partial t)_{c}
$$

The index $v$ in (25) refers to processes which are simulated both by the mesoscale model without convective forcing terms and by the cloud model, as for example, the vertical advection by the mesoscale vertical velocity

$$
\left(\frac{\partial X}{\partial t}\right)_{\mathrm{v}}=-w_{i j k}\left(\frac{\partial X}{\partial z}\right)_{i j k}=\left(X_{i j k}^{*}-X_{i j k}\right) / \Delta t
$$

where the last equality defines $X_{i j k}^{*}$ as the value, at time $t_{n+1}$, that these vertical processes would produce alone. The index LS in (25) refers to large-scale processes which are not at all simulated by the cloud model, for example horizontal advection.

Therefore, the new value, at time $t_{n+1}$, of the variable $X$ predicted by the mesoscale model under all processes which are included in the cloud model is:

$$
X^{n}+\Delta t\left[\left(\frac{\partial X}{\partial t}\right)_{\mathrm{v}}+\left(\frac{\partial X}{\partial t}\right)_{\mathrm{c}}\right]=X_{i j k}^{*}+F_{X} \Delta t
$$

in the grid mesh centered at the point of coordinates $\left(x_{i}, y_{j}, z_{k}\right)$. This value must be equal to the mean value $X_{i j k}^{c}$ that the cloud model predicts, at time $t_{n+1}$, in the same grid mesh:

$$
\begin{aligned}
X_{i j k}^{c}= & \int_{z k-\Delta k-1 / 2}^{z_{k}+\Delta_{k} / 2}\left\{\left[S-\sum_{N}\left(\sum_{\alpha} A_{i j \alpha}\right)_{N}\right]^{+} X_{i j k}^{*}\right. \\
+ & \left.\sum_{N}\left(\sum_{\alpha} A_{i j \alpha} X_{\alpha}{ }^{n+1}\right)_{N}\right\} d z / \int_{\{}\left\{S-\sum_{N}\left(\sum_{\alpha} A_{i j \alpha}\right)_{N}\right]^{+} \\
& \left.+\sum_{N}\left(\sum_{\alpha} A_{i j \alpha}\right)_{N}\right\} d z
\end{aligned}
$$

where $S=\Delta x \Delta y$ is the area of the mesh section and $(\cdot)^{+}=\max (\cdot, 0)$ represents the area of the mesh section not covered by any cell and in which the value $X_{i j k}^{*}$ is used to complete the integral.

The requirement that both models predict, after each time step $\Delta t$, the same mean value of the variable $X$ under the influence of processes which are included in the cloud model leads to the equality between (28) and (29), from which the convective forcing term $F_{X}$ can be evaluated as

$$
F_{X}=\left(X_{i j k}^{c}-X_{i j k}^{*}\right) / \Delta t .
$$

When $X=\rho \mathbf{u}, X=\rho C_{p d} T-L_{v} q_{c}$ and $X=q_{s}+q_{m}$ $=q_{v}+q_{c}$, the convective forcing terms $\rho \mathbf{F}_{c}, \rho Q_{c}$ and $S_{c}$, which are defined in Section 2, result from (30) respectively.

Note that the evaluation of the convective forcing terms involves only the vapor density and not the mass per unit volume of the rain drops or of graupel. This results from the assumption, made in the mesoscale model, that evaporation occurs whenever liquid water or ice appears in subsaturated air. If the mass of rain drops or graupel were included in the definition of the variables $X$ used to compute the convective forcing terms, then evaporation of these quantities would immediately cool the air in the mesoscale model, in opposition to the fact that a cloud constitutes a reserve of condensed water which evaporates slowly during its dissipation stage.

The mesoscale time step $(\Delta t=20 \mathrm{~s})$ is small compared to the life cycle of a cloud, so that the respective times of occurrence of the different processes and their spatial locations are of fundamental importance in the evaluation of the coupling terms. Thus, the time evolution is crucial in the evaluation of the convective forcing terms.

\section{d. Illustration of the coupling results}

As the cloud develops, it induces vertical fluxes of mass, energy and momentum in the mesoscale flow. As a result, a nonhydrostatic pressure field is modified in such a way that the resulting vertical gradient opposes the cloud development (Marwitz, 1973; Ramond, 1978; Klemp and Wilhelmson, 1978). This is illustrated in Fig. 5, which shows a vertical cross section of the perturbation pressure field $P_{i j k}^{\prime}$ obtained at 8 min of a simulation in which a cloud is created at $t=0$ in the cross-section plane and at $x=55 \mathrm{~km}$. During these $8 \mathrm{~min}$, the cloud rises up to $3.7 \mathrm{~km}$ while influencing the mesoscale fields. As a consequence, a high pressure dome appears centered at $x$ $=55 \mathrm{~km}$ just below the altitude of $4 \mathrm{~km}$, and a low pressure core is visible below $3 \mathrm{~km}$ at the same position. The resulting vertical pressure gradient at the cloud-top altitude is positive and opposes the cloud-top development. On the other hand, near the ground, the vertical pressure gradient is negative and favors the cloud development (Marwitz, 1973) by 
pulling negatively buoyant air parcels to the condensation level.

Another feature revealed in Fig. 5 is the slight dissymmetry of the perturbation pressure field near the cloud. This effect results from the convective transport of horizontal momentum, which induces values of the horizontal velocities that differ inside and outside the cloud (Ramond, 1978). Zones of horizontal convergence or divergence develop at the edge of the cloud and the pressure field is modified accordingly. This effect is illustrated in more detail in Section 5.

These coupling procedures between the cloud model and the mesoscale model produce expected interactions and allow the introduction of a nonhydrostatic pressure gradient into the quasi-one-dimensional cloud model; Holton (1973) has shown the importance of this introduction.

\section{e. Initial conditions for a new cell}

The different cells evolving in the mesoscale domain are created sequentially when the following creation criteria are satisfied at every time step, each horizontal position is tested and only one cloud can be created if; at first, the local vertical profile of the atmosphere is potentially unstable. This is quantified by the value of the CAPE (Convective Available Potential Energy) as defined by Moncrieff (1981) by

$$
\mathrm{CAPE}=g \int_{0}^{Z_{M}}\left(T_{v}^{\prime}-T_{v}\right) / T_{v} d z
$$

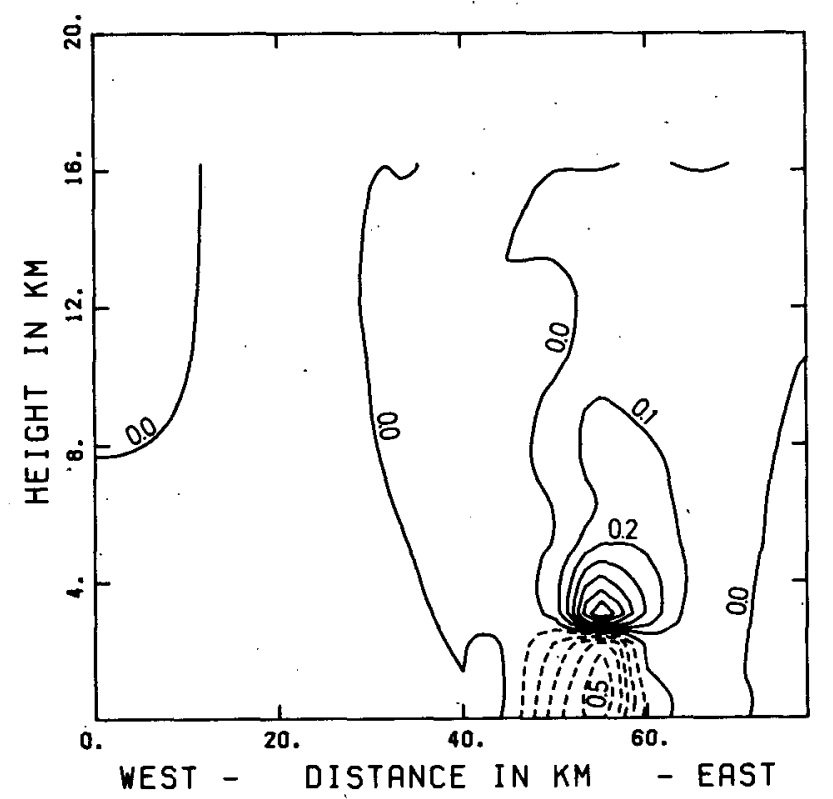

FIG. 5. Vertical cross section of the perturbation pressure $P^{\prime}$ (in $\mathrm{mb})$ made at $8 \mathrm{~min}$ of a simulation in which a cloud is created at $t=0$ and at the position $(x=55 \mathrm{~km}, y=80 \mathrm{~km})$. By the time of $8 \mathrm{~min}$, the cloud top reaches $3.7 \mathrm{~km}$. Isolines are drawn every 0.1 $\mathrm{mb}$ and dashed isolines correspond to negative values. where $T_{v}$ is the virtual temperature of the environment and $T_{v}^{\prime}$ that of a parcel rising adiabatically from the ground. The term $Z_{M}$ is the altitude where, above the condensation level, $T_{v}^{\prime}$ becomes smaller than $T_{v}$. The value of CAPE must be larger than $1500 \mathrm{~m}^{2} \mathrm{~s}^{-2}$ according to Pastushkov (1973). Second, the chosen point must be where the horizontal humidity convergence is maximum (Hudson, 1971) and is above a threshold value:

$$
\operatorname{div}\left(q_{v} \mathbf{u}\right)<d_{0}<0 .
$$

When both conditions are satisfied, a new cell is created. In fact, the threshold value $d_{0}$ is the controlling factor of the amount of convection. The more negative this value is, the less often a new cell is created since perturbations of the mesoscale fields build up gradually. When the creation of a new cell is accepted, its initial conditions are as follows:

- the cloud top height is taken just above the condensation level;

- the vertical velocity in the internal cylinder is taken as a constant $\left[w_{1}\left(z, t_{i}\right)=2 \mathrm{~m} \mathrm{~s}^{-1}\right]$;

- the radii of the three cylinders are arbitrarily imposed by lack of a relationship which could predict them $\left(R_{1}=2200 \mathrm{~m}, R_{2}=3300 \mathrm{~m}, R_{3}=9900 \mathrm{~m}\right)$;

- the humidity is increased in the internal cylinder under the condensation level. It should be noted that during the cloud development the boundary condition for the humidity remains unmodified so that the increase of humidity is restricted to the initial value (paragraph 3-8).

These initial conditions and the above creation criteria may appear quite arbitrary, but they are, for the most part, compatible with the poor knowledge we have on the conditions that prevail when a new cloud starts developing.

\section{Simulation results and comparison with experi- mental data}

Several simulations have been made in order to test the sensitivity of the organization mechanisms to the environmental parameters; this study is described in a Part II, to be published later. In the present Part, we only describe the main features of the flow obtained during one simulation in which several clouds developed. Comparisons of these features are also made with some of the characteristics of a squall line that can be deduced from an observational field program (Sommeria and Testud, 1984). The observational network covers an area of $50 \mathrm{~km} \times 50 \mathrm{~km}$ and includes two Doppler radars, an acoustic sounder and a central meteorological station equipped for rawin sounding and interrogation of remote ground meteorological stations.

The initial conditions for the mesoscale model in this simulation are deduced from rawinsonde data 
obtained at 1500 GMT 21 June 1981, almost 13 hours before the squall line passed through the experimental area near Korhogo (Ivory Coast). The initial conditions are spatially uniform; the vertical profiles of the temperature, dew-point temperature and horizontal wind are drawn in Fig. 6 in a skew T diagram. A heat source is introduced before the real start of the simulation in order to create a zone of maximum convergence in the middle of the domain $(x=80 \mathrm{~km}, y=80 \mathrm{~km})$. At $t=0$ a first cloud is created at this point, and as the simulation proceeds a total of 19 cells are created in a time of $23 \mathrm{~min}$ while the total simulation time is $54 \mathrm{~min}$. The maximum number of cells is presently limited to 20 in the program. This small number of cells limits the convective activity in this example, so that steady state cannot be achieved.

\section{a. Dynamical and thermodynamical fields at the ground}

The cell trajectories are drawn in Fig. 7 in a horizontal cross section of the mesoscale domain. Figure 7 shows that the cells move towards the north,

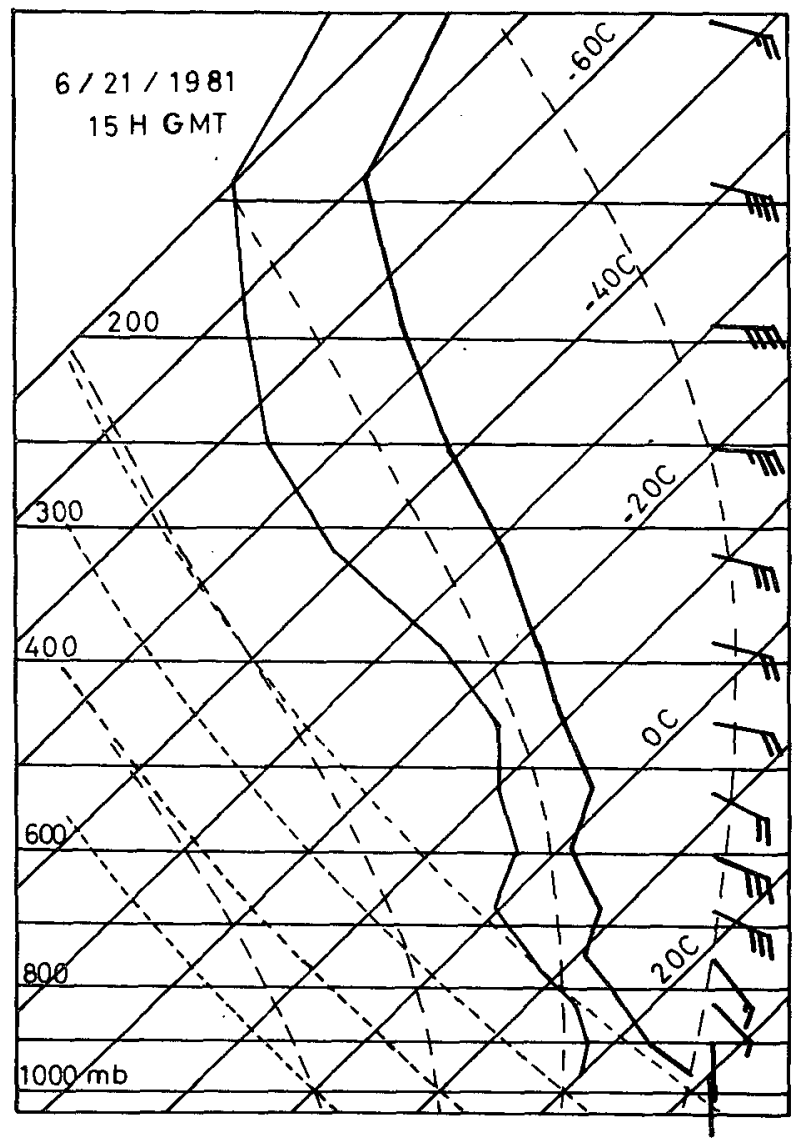

FIG. 6. Skew- $T$ diagram showing the temperature, dew-point temperature and velocity vector as function of the pressure. i.e. towards the top of the figure, and then towards the northwest direction. Their position at $t=40 \mathrm{~min}$ of the simulation, which is the time at which the mesoscale fields are described later, is indicated by the center of the dashed circles of radius $3 \mathrm{~km}$ that schematize the cloud extent. The numbers on the edge of the trajectories indicate the order in which the clouds are created. A close examination of this figure suggests that the new clouds are mainly created west to northwest of the oldest ones.

The main reason for this preferred area of creation stems from the fact that a zone of maximum convergence of the horizontal wind at the ground exists, west of the convective cells, in the mesoscale domain. [The creation criteria (Section 3) imply that new cells are created in this zone.] This zone is visualized in the plot of the horizontal wind vectors shown in Fig. 8 for the first level of the model and at $40 \mathrm{~min}$ of the simulation. The horizontal winds are rather weak except in a circular zone, later referred to as the perturbation region, in which all the convective cells are situated at that time, as shown in Fig. 7 . In this perturbation region, the winds are rather uniform and directed to the west-northwest. This velocity field implies that a convergence zone exists on the west side of the perturbation region, as discussed above, while a divergence zone is created on the east side.

The larger values of the horizontal winds in the perturbation region result from the downward transport of horizontal momentum caused both by the mean mesoscale subsidence motion surrounding the convective cells due to negative buoyancy effects and by the local convective downdrafts driven by precipitation loading and evaporational cooling in the intermediate cylinder of each cell. This last effect is introduced in the mesoscale equations by the convective forcing terms (Section 4).

The comparison of the horizontal fields at different simulation times indicates that the perturbation region keeps its characteristics during the second half of the simulation while increasing its east-west size as it propagates in the western direction. Indeed, between 40 and $52 \mathrm{~min}$, the perturbation region is displaced by about $8.6 \mathrm{~km}$, leading to a mean velocity of about $12 \mathrm{~m} \mathrm{~s}^{-1}$. As a comparison, the observed squall line has a mean velocity of $19 \mathrm{~m} \mathrm{~s}^{-1}$ towards the westsouthwest direction and the squall front, in which most convective motions develop, makes a northsouth arch of more than $600 \mathrm{~km}$ long (Fig. 7 in Sommeria and Testud, 1984).

Despite the horizontal extent of the perturbation region being only about $60 \mathrm{~km}$ in the simulation, the mesoscale fields are very similar in the squall front region, as can be deduced from the comparison of the horizontal cross sections of the pressure perturbation at the ground. Isolines of the simulated perturbation pressure are drawn in Fig. 9 at the ground 


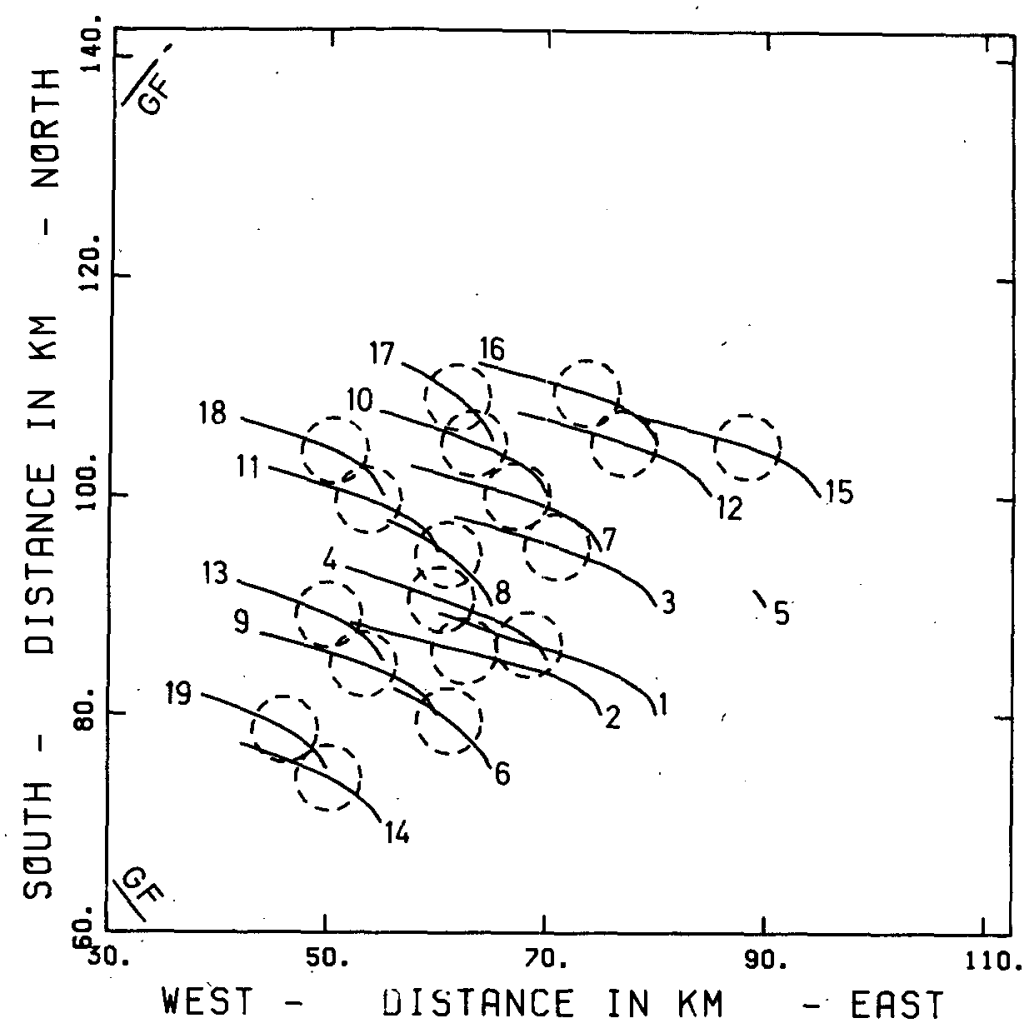

Fig. 7. The cell trajectories are shown by the solid line (from right to left as time increases) on the horizontal cross section of the mesoscale domain. The position and extent of the cells at $40 \mathrm{~min}$ of the simulation are indicated by the dashed circles. The numbers at the edge of the trajectories indicate the order in which the cells have been created.

and at 40 min of the simulation while Fig. 10 shows isolines of the measured perturbation pressure field over a limited area of the squall line. Figure 10 is deduced from ground station measurements assuming that the squall line moves over the experimental area without much change during less than one hour so that the "steady state" assumption is used to transform temporal data into spatial data. In both figures, the pressure increases in the west side of the perturbation region (ahead of it) up to $2 \mathrm{mb}$ inside an elongated north-south region of $20 \mathrm{~km}$ width. In response to this pressure increase, the wind velocity increases strongly so that the region is usually called the gust front of the squall line. Behind it, the pressure decreases in a much wider region.

The time evolution of the meteorological parameters, recorded by one of the ground stations, is shown in Fig. 11. Assuming a steady-state propagation of the squall line, a distance scale may be added to the time scale, taking into account the propagation speed of $19 \mathrm{~m} \mathrm{~s}^{-1}$. Figure 11 shows that while the wind speed increases at the same time as the pressure does, the first precipitations occur only $10 \mathrm{~km}$ behind the gust front. This feature is also simulated by the model; comparison of Figs. 7 and 9 indicates that the closest convective cells are more than $25 \mathrm{~km}$ away from the gust front. This larger value is partly due to the poor resolution of the mesoscale model $(5 \mathrm{~km})$, which smoothes out sharp discontinuities such as the gust front shown in Fig. 11.

Another characteristic of the squall front shown in Fig. 11 is the decrease of temperature and relative humidity almost $7 \mathrm{~km}$ behind the gust front and 3 $\mathrm{km}$ before the first precipitations. This temperature decrease indicates that the gust front is the edge of a gravity current that is generated by the squall line. Just after its small decrease, the relative humidity increases almost to saturation due to the decrease of temperature and the increase of mixing ratio induced by precipitation evaporation. The decrease of temperature a few kilometers behind the gust front is also simulated by the model, as shown in Fig. 12, in which a horizontal cross section of the temperature field is made at the ground and at $40 \mathrm{~min}$ of the simulation. It should be noted, however, that the magnitude of this decrease is larger in the squall line $(3 \mathrm{~K})$ than in the simulated perturbation region $(0.2$ $\mathrm{K})$. This discrepancy partly results from ground temperatures being kept constant in the constant flux layer formulation (Nickerson and Smiley, 1975), 


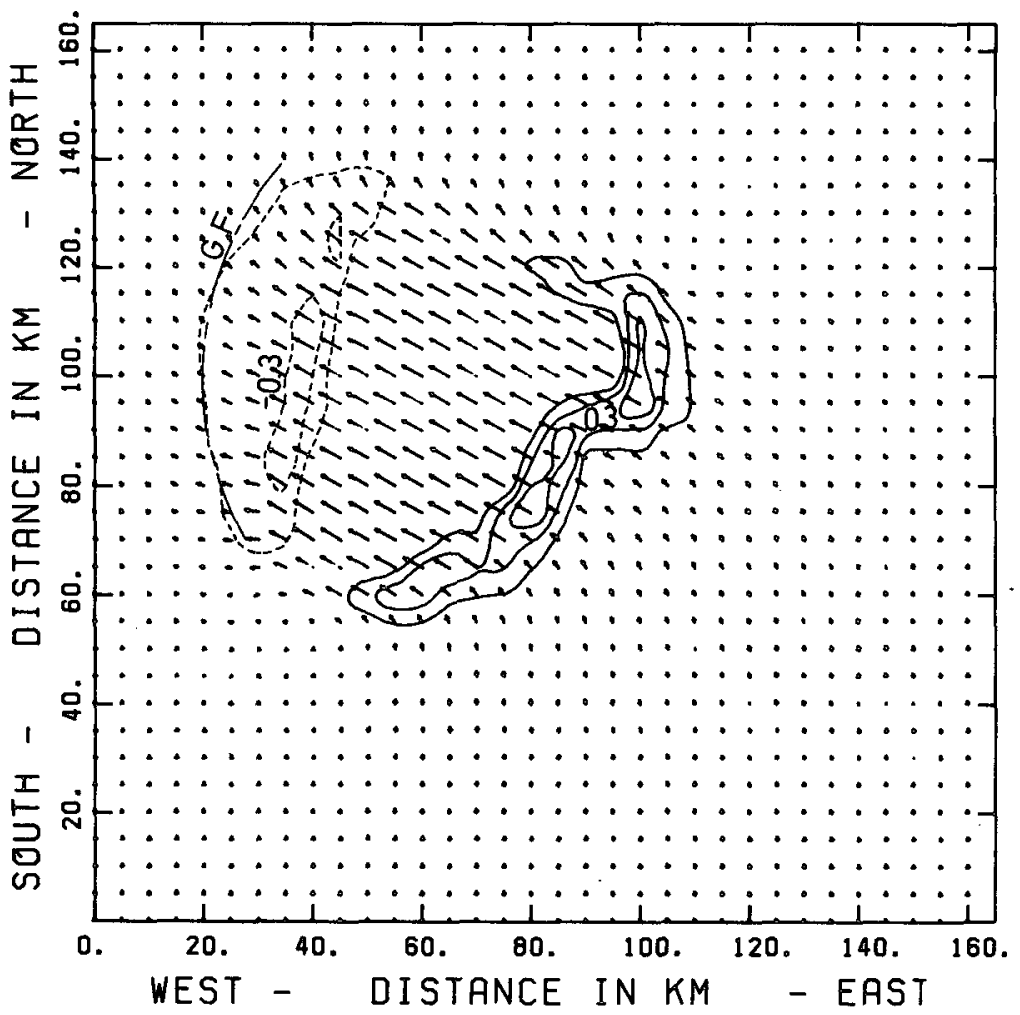

Fig. 8. Horizontal cross section of the horizontal wind (arrows) at the first level at $40 \mathrm{~min}$ of the simulation. The arrows show the path of a parcel, having the air velocity, during a $500 \mathrm{~s}$ travel. The dash-dotted arc indicates the gust front (GF) position. Isolines of the divergence of the horizontal wind are also shown (intervals are $0.15 \times 10^{-3} \mathrm{~s}^{-1},>0$ divergence, $<0$ convergence).

causing the layer to become unstable when the above air cools, so that the ground heats up the air too much. This feature implies that the simulated flow can bear more resemblance to ocean squall lines (Zipser, 1977). The smaller temperature decrease partly explains the small propagation speed which has been simulated $\left(12\right.$ versus $19 \mathrm{~m} \mathrm{~s}^{-1}$ for the observed value) since the strength of the gravity current, which generates the gust front, is decreased.

Comparison of the observed and simulated fields at the ground shows that the relative position of the different changes are well simulated by the model even if their magnitudes are not all well predicted. Some of the other main causes of the discrepancies are the limited extent of the perturbation region and the fact that no new cells are created after $23 \mathrm{~min}$ of the simulation while the perturbation region still propagates faster than the cells and therefore moves away from the closest ones. These restrictions are now being removed in simulations in which the maximum number of cells is increased.

\section{b. Vertical structure of the dynamical fields}

The three-dimensional flow is visualized in Fig. 13, which shows a vertical cross section of the relative velocity field made along an east-west direction through the middle of the perturbation region ( $y$ $=105 \mathrm{~km}$ ) at $40 \mathrm{~min}$ of the simulation. The relative wind is obtained by substracting the propagation velocity of the perturbation region $\left(u=12 \mathrm{~m} \mathrm{~s}^{-1}\right)$. The arrows in Fig. 13 indicate the path of a parcel moving during $500 \mathrm{~s}$ with the relative air velocity at each grid point.

Figure 13 indicates that, in the lowest layers, air enters the perturbation region in the front part (west side). The relative velocity decreases across the gust front and becomes minimum inside the perturbation region. As it slows down, the air rises above the gravity current $(x=40 \mathrm{~km})$ and, a few kilometers behind, it rises up to $14 \mathrm{~km}$ in a narrow updraft. In this particular cross section, this updraft is induced by the convective cell No. 18 which is situated there, at this time, as shown in Fig. 7. This velocity field results from the fact that the new cells are created just behind the gust front in a zone of maximum convergence associated with mesoscale upward motions, and that by the time $(20 \mathrm{~min})$ these cells reach the highest level, the gust front has moved a few kilometers ahead of them. Older cells can induce weaker vertical motions such as the updraft, shown 


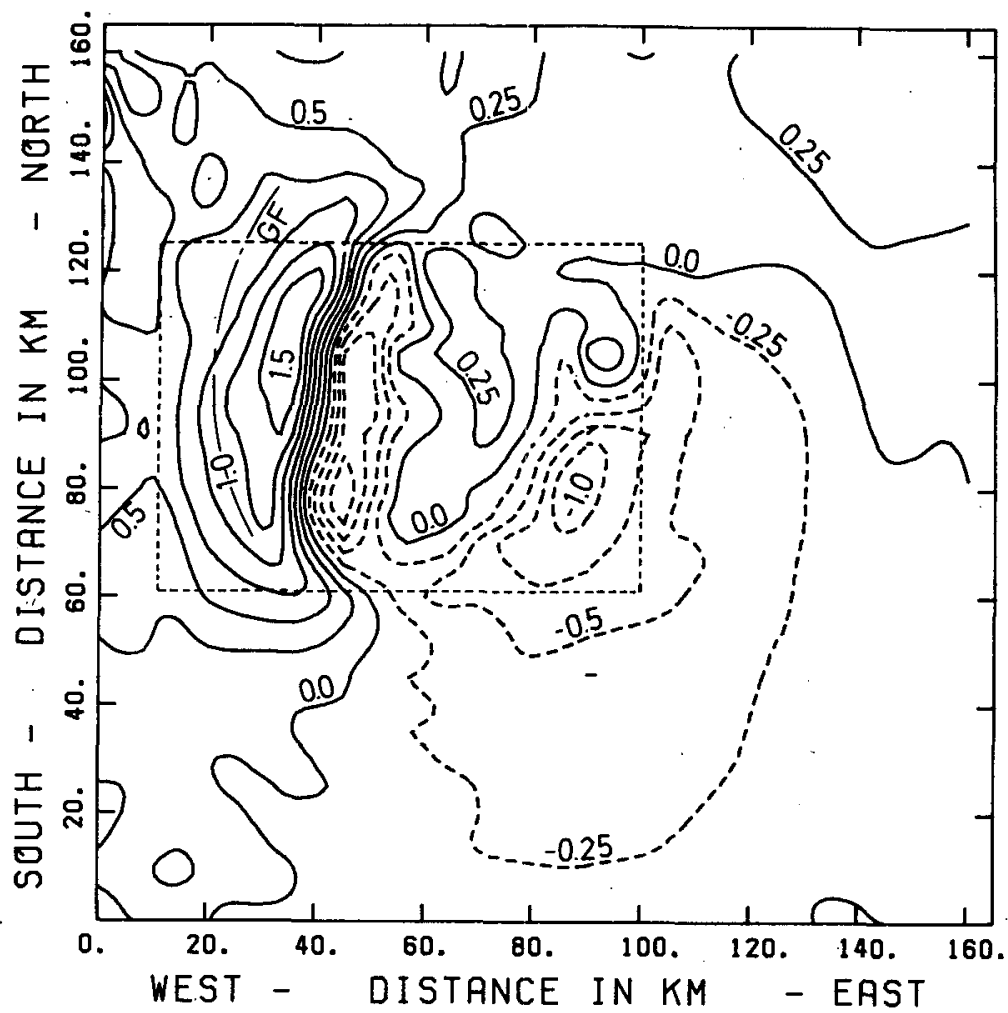

FIG. 9. Horizontal cross section of the ground perturbation pressure $P^{\prime}$ (in $\mathrm{mb}$ ) made at $40 \mathrm{~min}$ of the simulation. Isolines are drawn every $0.25 \mathrm{mb}$; dashed isolines correspond to negative values. The dash-dotted arc indicates the gust front (GF) position. The dotted rectangle indicates an area of the same extent as in Fig. 10.

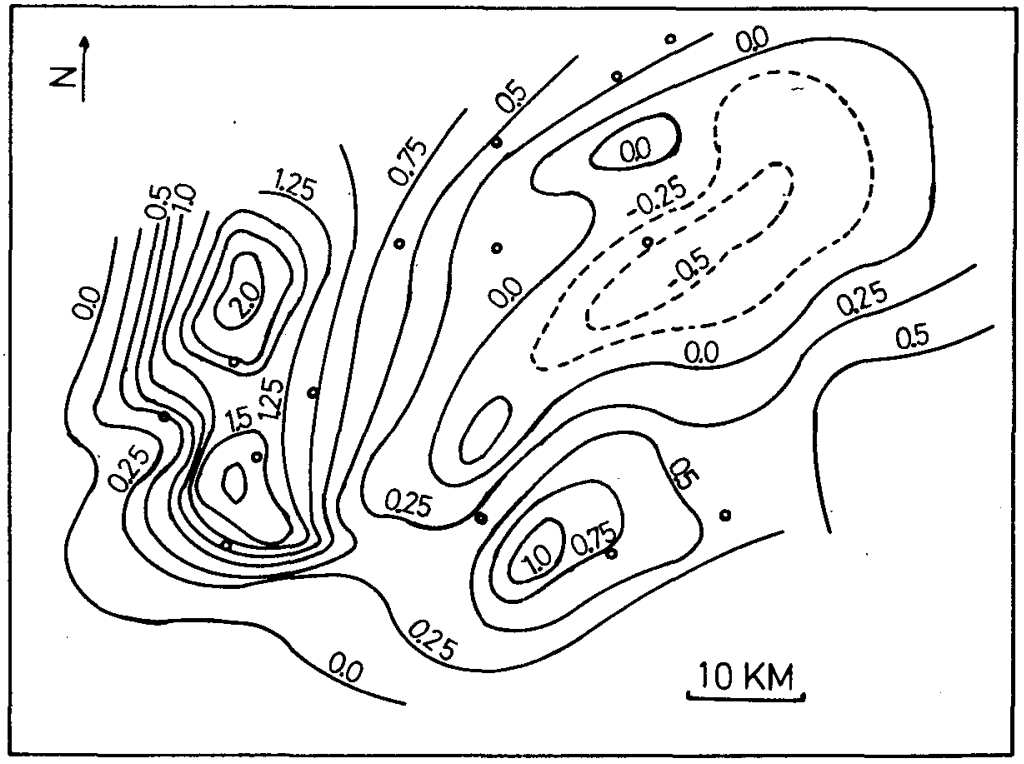

FIG. 10. Horizontal cross section of the ground perturbation pressure (in mb) deduced from the measurements made every $30 \mathrm{~s}$ by the 13 ground stations (indicated by circles). This cross section is derived under the assumption that the squall line does not evolve substantially during its motion with a velocity of $19 \mathrm{~m} \mathrm{~s}^{-1}$ in the west-southwest direction. A long-term tendency has been removed from the pressure values. 


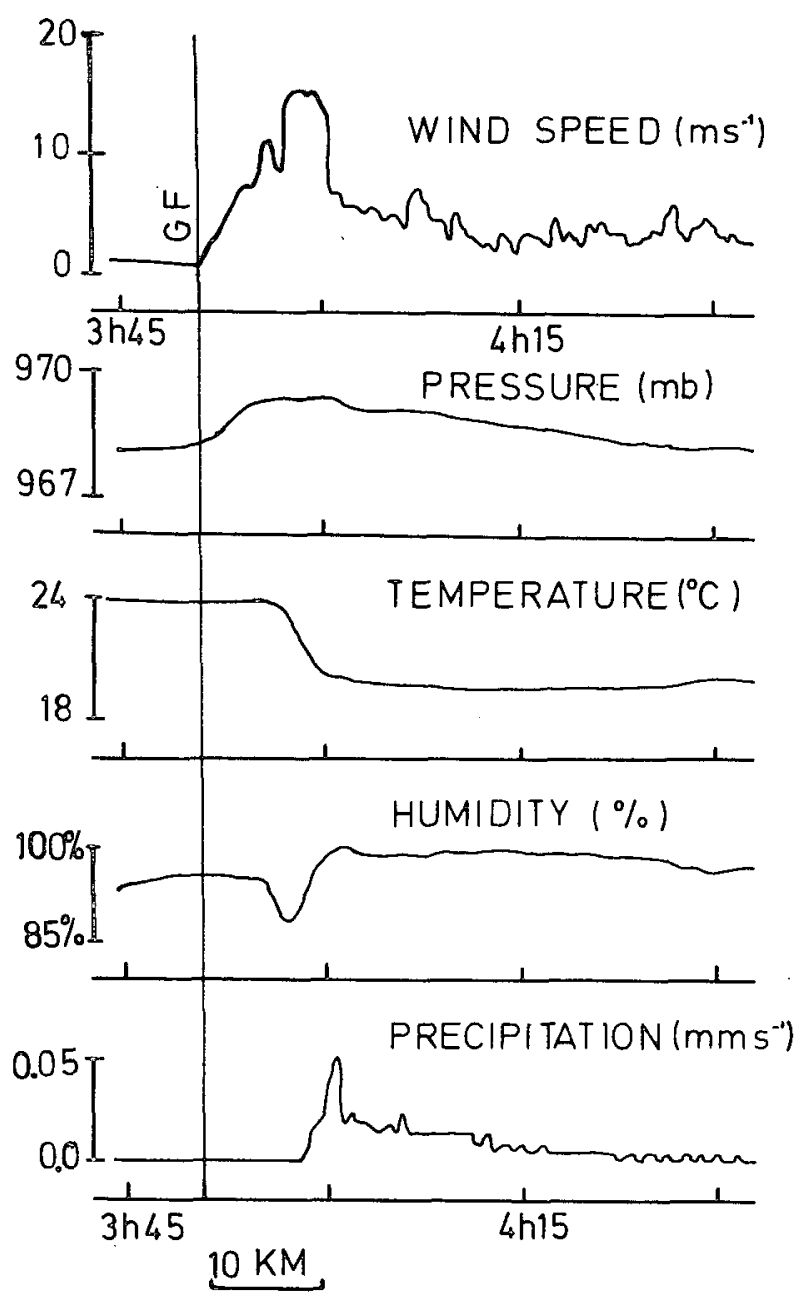

FIG. 11. Time evolution of the meteorological parameters, measured by the ground station situated in the negative perturbation pressure area in Fig. 10, every $30 \mathrm{~s}$ during the squall-line crossing.

at $x=90 \mathrm{~km}$ in Fig. 13, which is created by the cell No. 15 (Fig. 7). These oldest cells also constitute reserves of liquid water that partly precipitate and partly evaporate, modifying the flow conditions in the rear part of the perturbation region. In the rear part, localized downdrafts develop between convective cells and participate in the vertical transport of horizontal momentum. As a consequence, the vertical gradient of the horizontal velocity is smaller inside the perturbation region (55 $\mathrm{km}<x<80 \mathrm{~km}$ ) than ahead of it $(x<30 \mathrm{~km})$.

The downdraft shown in Fig. 13 for $x=105 \mathrm{~km}$ and at altitudes near $8 \mathrm{~km}$ is created by a zone of convergence of the horizontal wind (as shown by the isocontours) at $12 \mathrm{~km}$ altitude and by a zone of divergence near $6 \mathrm{~km}$. These zones result from the different values of the horizontal wind inside and outside the perturbation region in the near part. On the other hand, a divergence zone appears at $12 \mathrm{~km}$ altitude and a convergence zone near $6 \mathrm{~km}$ in the front part of the perturbation region $(x=40 \mathrm{~km})$. The resulting vertical motion is upward and is favorable to the cloud development.

In the case of squall lines, the air behind the squall front has been perturbed by stratiform clouds over distances of hundreds of kilometers since the squall front moves, in general, faster than the air at midlevels. The conditions behind the squall front are important to the squall-line dynamics (Lilly, 1979; Le Mone, 1983), and these effects are not completely reproduced in the simulation due to its limited duration.

\section{Summary and conclinsions}

A new model is proposed for the simulation of mesoscale flows perturbed by deep convective clouds. It is based on the time-dependent coupling between a nonhydrostatic three-dimensional mesoscale model and a quasi-one-dimensional cloud model. Each model simulates processes with particular characteristic length scales $(10-200 \mathrm{~km}$ for the mesoscale model and $0.5-10 \mathrm{~km}$ for the cloud model) not explicitly simulated by the other one. The coupling terms transmit to each model the influence of processes described by the other one. On the one hand, the local evaluations of the environmental conditions of each cell and of the nonhydrostatic pressure profile transmit, to the cloud model, the influence of the mesoscale dynamical and thermodynamical fields. On the other hand, the convective forcing terms, evaluated from the cloud model, transmit to the mesoscale model the effects of the microphysical processes. The interactions between the convective cells and the mesoscale flow appear to be simulated in a realistic manner since a mesoscale pressure field develops in response to a cell growth.

A particular simulation is analyzed. Analysis shows that the main characteristics of the flow perturbed by convective cells are similar to those of a squall line deduced from ground station measurements. In the simulation, a gust front develops ahead of a perturbation region in which the horizontal velocity changes in response to vertical transport of horizontal momentum due to convective motions. The pressure increases across the gust front, and $7 \mathrm{~km}$ behind it the temperature decreases while the pressure reaches a maximum value. The relative position of these different effects is well simulated by the model even though their magnitudes are sometimes poorly estimated. The main discrepancies result from the limited number of cells that have been simulated, from the short duration of the simulation and from the poor spatial resolution $(5 \mathrm{~km})$ of the mesoscale model. Improvements of the models are underway such as a possibility of moving the reference frame at a constant velocity with respect to the ground; a modification of the upstream vertical advection term at the first level 


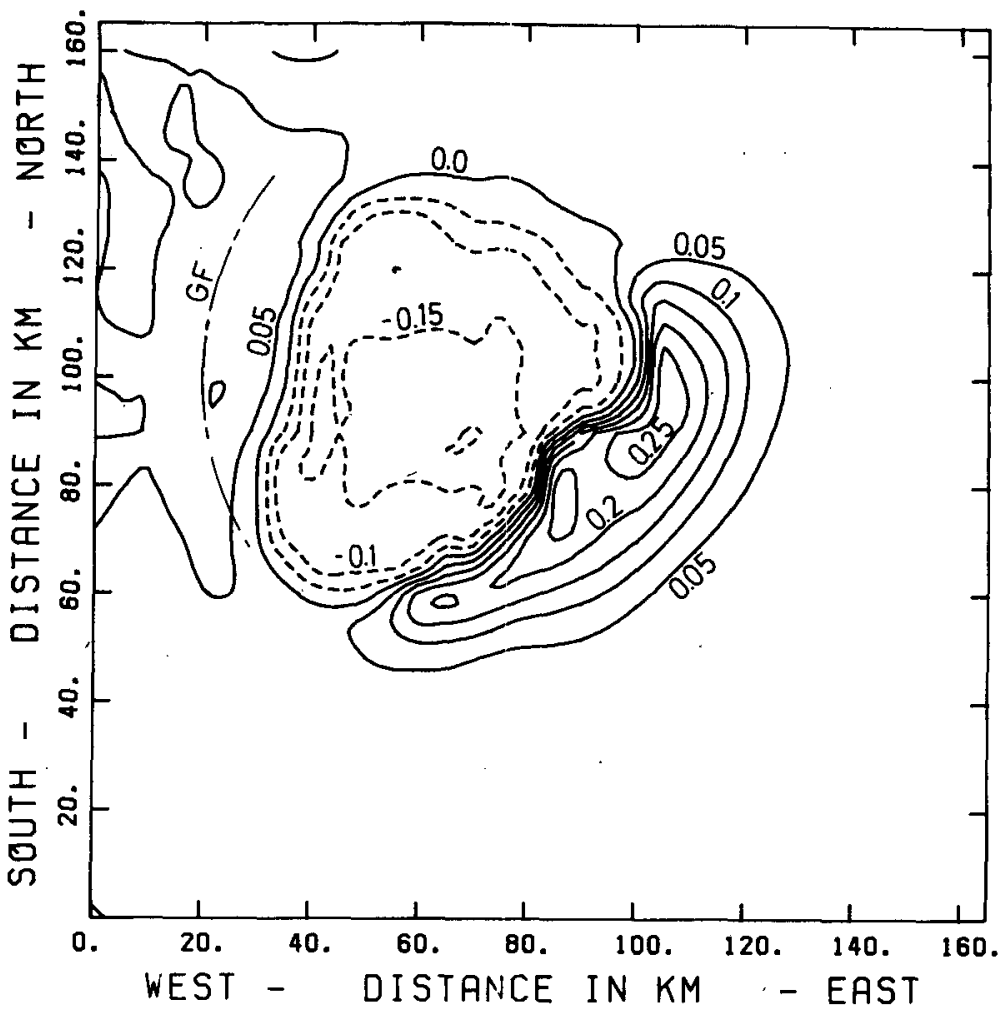

Fig. 12. Horizontal cross section of the temperature at the ground at $40 \mathrm{~min}$ of the simulation. Isolines are drawn every $0.05^{\circ} \mathrm{C}$; dashed isolines correspond to temperatures below the initial value.

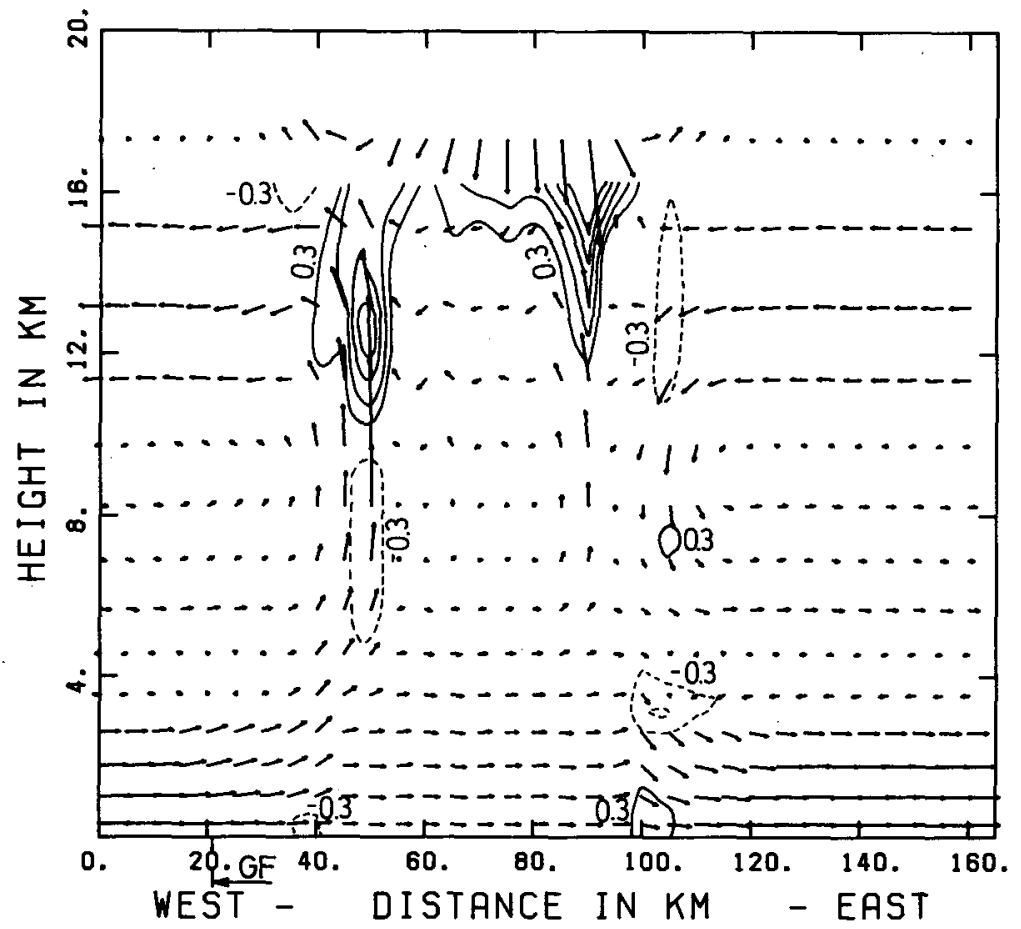

FIG. 13. East-west vertical cross section of the wind relative to the perturbation region made at $y=105 \mathrm{~km}$ at $40 \mathrm{~min}$ of the simulation. Isolines of the divergence of the horizontal wind are also drawn (intervals are $0.3 \times 10^{-3} \mathrm{~s}^{-1},>0$ divergence, $<0$ convergence). 
(M. Miller; personal communication, 1984); and a slight modification of the pressure equation at the top which would avoid the increase in vertical velocity at the top or in the mean pressure at the ground.

This model can serve as a tool to be used in the studies of such questions as:

- What are the mesoscale factors that are favorable or unfavorable to a mesoscale organization of convective clouds (potential instability, vertical gradient of the horizontal wind, low level jet, and so on)?

- What happens when several mechanisms (vertical transport of horizontal momentum, pressure gradients created by convective motions, by divergence or convergence of the horizontal winds, and so on) have opposite effects on the organization process?

- Could a squall line develop in a given environment?

This tool can be very useful since simulations are not very expensive and the initial vertical profiles of temperature, humidity and wind speed and direction can easily be varied. A simulation with 20 clouds takes $21 \mathrm{~min}$ on an IBM $370-168$ for a simulated time of $54 \mathrm{~min}$.

Acknowledgments. The author is indebted to Doctor H. Isaka for his help and guidance in this work and to Professors R. G. Soulage and R. Rosset for their encouragement and support. All the encouragement and advice received during this work have been appreciated. The work of B. Pinty in providing the experimental data and the corresponding figures is gratefully acknowledged. The author is grateful to the referees for their help in improving the manuscript. Many thanks are due to R. Pejoux and to Miss J. Duron for their excellent computer assistance and to Mrs. C. Paquet and J. Squarise for their dedicated typing and editing of the manuscript. This work has been partially supported by the "ATP Recherches Atmosphériques" committee (Institut National d'Astronomie et de Géophysique).

\section{APPENDIX}

\section{Derivation of the Discretized Helmholtz Equation}

As in Tapp and White (1976), the momentum equation (5) is discretized according to (9), with the first two terms in the right-hand side evaluated as the mean value between time levels $n$ and $n+1$. The following equation results:

$$
\mathbf{u}^{n+1}=\mathbf{u}^{*}-\Delta t \nabla \pi /(2 \bar{\rho})-g \mathbf{k} \Delta t \pi /(2 \gamma \bar{P})
$$

where the vector $\mathbf{u}$ can be evaluated from the values of the fields at time level $n$ :

$$
\begin{aligned}
\mathbf{u}^{*}=\mathbf{u}^{n}+\Delta t[-\mathbf{u} & \cdot \nabla \mathbf{u}-\nabla P^{\prime} / \rho \\
& \left.-g \mathbf{k}\left(\rho^{n}-\bar{\rho}\right) / \rho^{n}-\mathbf{f} \times \mathbf{u}+\mathbf{F}\right]
\end{aligned}
$$

in which the superscript $n$ has been omitted. The pressure increment $\pi$ which appears in the last two terms in (A1) is defined by:

$$
\pi=P^{n+1}-P^{n} \text {. }
$$

The last two terms in (A2) are correction terms which are due to the semi-implicit method of integration; namely they arise from the mean value between time levels $n$ and $n+1$ in the pressure gradient term and in the buoyancy term respectively. The discretized pressure tendency equation (6) involves, by the semiimplicit method, the new value of the velocity $a^{n+1}$ which is obtained from (A1). The following Helmholtz equation for the pressure increment $\pi$ results:

$$
\begin{aligned}
\Delta \pi-\frac{\partial \pi}{\partial z} \frac{\partial \ln \bar{\rho}}{\partial z} & \\
& -\pi\left(\frac{4}{\Delta t^{2} C_{s}^{2}}-\frac{(\gamma-1) g^{2}}{C_{s}^{4}}\right)=G^{n},
\end{aligned}
$$

where $C_{s}(z)=\left(\gamma R \bar{T}(z) / M_{d}\right)^{1 / 2}$ is the speed of sound at the altitude $z$ and $G^{n}$ is given in terms of the field values at time level $n$ by:

$$
\begin{aligned}
G^{n} & =2 \bar{\rho} \operatorname{div}\left(\mathbf{u}^{n}+\mathrm{u}^{*}\right) / \Delta t-\left[2 g \bar{\rho}\left(w^{n}+w^{*}\right)\right. \\
& \left.-4 \gamma P^{\prime n} \operatorname{divu}-4 \mathbf{u}^{n} \cdot \nabla P^{\prime n}+4 \rho(\gamma-1) Q\right] /\left(\Delta t C_{s}^{2}\right)
\end{aligned}
$$

where $Q$ includes both the convective forcing heat source $Q_{c}$ and the mesoscale heat source $Q_{L}$ due to the condensation resolved on the mesoscale grid. This last term provides a coupling between the dynamical equations and the thermodynamical equation (7). This coupling is avoided by using in (A5) an estimated value of $Q_{L}$ deduced from the vertical velocity and from the water vapor deficit $q_{m}$. The right-hand side of the Helmholtz equation (A4) is computed from (A5) in which $\mathrm{u}^{*}$ is evaluated from (A2). The Helmholtz equation is solved by a direct numerical method based, as in TW, on the diagonalization of the matrix representing the finite difference of the vertical differential operator. The resulting two-dimensional Helmholtz equations are solved by a cyclic reduction algorithm.

The new value of the pressure is deduced from (A3) and the new value of the velocity from (A1). The thermodynamical equations are integrated simultaneously from time level $n$ to time level $n+1$ using the mean values, between time levels $n$ and $n+1$, of the dynamical fields.

The final adjustment to the temperature and water vapor deficit is due to the condensation process and gives the true value of $Q_{L}$. However, since most condensation occurs in the convective cells, the mesoscale condensation is weak and $Q_{L}$ usually equals zero.

\section{REFERENCES}

Anthes, R. A., 1977: A cumulus parameterization scheme utilizing a one-dimensional cloud model. Mon. Wea. Rev., 105, 270286. 
Arakawa, A., and W. H. Schubert, 1974: Interaction of a cumulus cloud ensemble with the large-scale environment, Part I. $J$. Atmos. Sci., 31, 674-701.

Arakawa, A., and V. R. Lamb, 1977: Computational design of the basic dynamical processes of the UCLA general circulation model. Methods in Comp. Physics, Academic Press, 173-265.

Cotton, W. R., and G. J. Tripoli, 1978: Cumulus convection in shear flow-Three-dimensional numerical experiments. $J$. Atmos. Sci., 35, 1503-1521.

Fritsch, J. M., and C. F. Chappell, 1980a: Numerical prediction of convectively driven mesoscale pressure systems. Part I: Convective parameterization. J. Atmos. Sci., 37, 1722-1733.

- , and $-1980 \mathrm{~b}$ : Numerical prediction of convectively driven mesoscale pressure systems. Part II: Mesoscale model. J. Atmos. Sci., 37, 1734-1762.

Geleyn, J. F., C. Girard and J. F. Louis, 1982: A simple parameterization of moist convection for large-scale atmospheric models. Contrib. Atmos. Phys., 55, 325-334.

Holton, J. R., 1973: A one-dimensional cumulus model including pressure perturbations. Mon. Wea. Rev., 101, 201-205.

Hudson, H. R., 1971: On the relationship between horizontal moisture convergence and convective cloud formation. $J$. Appl. Meteor., 10, 755-762.

Kessler, F., 1969: On the Distribution and Continuity of Water Substance in Atmospheric Circulation. Meteor. Monogr. No. 10, Amer. Meteor. Soc., 84 pp.

Klemp, J. B., and R. B. Wilhelmson, 1978: The simulation of three-dimensional convective storm dynamics. J. Atmos. Sci., 35, 1071-1096.

Kreitzberg, C. W., and D. J. Perkey, 1976: Release of potential instability. Part I: A sequential plume model within a hydrostatic primitive equation model. J. Atmos. Sci., 33, 456-475.

Kuo, H. L., 1974: Further studies of the parameterization of the influence of cumulus convection on large-scale flow. J. Atmos. Sci., 31, 1232-1240.

Le Mone, M. A., 1983: Momentum transport by a line of cumulonimbuis. J. Atmos. Sci., 40, 1815-1834.

Lilly, D. K., 1979: The dynamical structure and evolution of thunderstorms and squall lines. Annu. Rev. Earth Planet. Sci., 7, 117-16i.

Lopez, R. E., 1973: A parametric model of cumulus convection. J. Atmos. Sci., 30, 1354-1373.

Marwitz, J. D., 1973: Nonhydrostatic pressures in severe thunderstorms. 8th Conf. on Severe Local Storms, Denver, CO, Amer. Meteor. Soc., 14-17.

Moncrieff; M. W., 1981: A theory of organized steady convection and its transport properties. Quart. J. Roy. Meteor. Soc:, 107, 29-50.

Nickerson, E. C., and V. E. Smiley, 1975: Surface layer and energy budget parameterizations for mesoscale models. J. Appl. Meteor., 14, 297-300.

O'Brien, J. J., 1970: On the vertical structure of the eddy exchange coefficient in the planetary boundary layer. J. Atmos. Sci., 27, 1213-1215.

Ogura, Y., and T. Takahashi, 1971: Numerical simulation of the life cycle of a thunderstorm cell. Mon. Wea. Rev., 99, 895911.

Orlanski, I., 1975: A rational subdivision of scales for atmospheric processes. Bull. Amer. Meteor. Soc., 56, 527-530.

- 1976: A simple boundary condition for unbounded hyperbolic flows. J. Comput. Phys., 21, 251-269.

Pastushkov, R. S., 1973: The effect of vertical wind shear on the development of convective cloud. Izv. Acad. Sci. URSS, Atmos. Oceanic Phys., 9, 5-11. Corrigendum. Quart. J. Roy. Meteor. Soc., 1976, 102, 264.

Pointin, Y., 1984a: Wet equivalent potential temperature and enthalpy as prognostic variables in cloud modeling. J. Atmos. Sci., 41, 651-660.

_- 1984b: Simulations numériques de quelques cas de convection organisée à l'échelle mésosynoptique: Application à la dynamique des lignes de grains. Thèse d'Etat, Université de Clermont II, France, $150 \mathrm{pp}$.

Ramond, D., 1978: Pressure perturbations in deep convection: An experimental study. J. Atmos. Sci., 35, 1704-1711.

Rosenthal, S. L., 1979: The sensitivity of simulated hurricane development to cumulus parameterization details. Mon. Wea. Rev., 107, 193-197.

Sommeria, G., 1976: Three-dimensional simulation of turbulent processes in an undisturbed trade wind boundary layer. $J$. Atmos. Sci., 33, 216-241.

for the study of dynamics and electrical activity of deep convection in continental tropical region. Bull. Amer. Meteor. Soc., 65, 4-10.

Tapp, M. C., and P. W. White, 1976: A non-hydrostatic mesoscale model. Quart. J. Roy. Meteor. Soc., 102, 277-296.

Tripoli, G. J., and W. R. Cotton, 1982: The Colorado State University three dimensional cloud/mesoscale model 1982. Part 1: General theoretical framework and sensitivity experiments. J. Rech. Atmos., 16, 185-219.

Wang, J. Y., 1983: A quasi-one-dimensional, time-dependent and nonprecipitating cumulus cloud model: On the bimodal distribution of cumulus cloud height. J. Atmos. Sci., 40, 651664.

Wilhelmson, R. B., and C. S. Chen, 1982: A simulation of the development of successive cells along a cold outflow boundary. J. Atmos. Sci., 39, 1466-1483.

Wisner, C., H. D. Orville and C. Myers, 1972: A numerical model of a hail-bearing cloud. J. Atmos. Sci., 29, 1160-1181.

Yau, M. K., and R. Michaud, 1982: Numerical simulation of a cumulus ensemble in three dimensions. J. Atmos. Sci., 39, $1062-1079$.

Zipser, E: J., 1977: Mesoscale and convective-scale downdrafts as distinct components of squall-line structure. Mon. Wea. Rev., $105,1568-1589$. 